\title{
Interplay between singing and cortical processing of music: a longitudinal study in children with cochlear implants
}

\author{
Ritva Torppa ${ }^{1,2 *}$, Minna Huotilainen ${ }^{1,2,3}$, Miika Leminen ${ }^{1,2,4}$, Jari Lipsanen ${ }^{5}$ and Mari Tervaniemi ${ }^{1,2}$ \\ ${ }^{1}$ Cognitive Brain Research Unit, Cognitive Science, Institute of Behavioural Sciences, University of Helsinki, Helsinki, Finland \\ ${ }^{2}$ Finnish Centre for Interdisciplinary Music Research, University of Helsinki, Helsinki, Finland \\ ${ }^{3}$ Brain Work Research Centre, Finnish Institute of Occupational Health, Helsinki, Finland \\ ${ }^{4}$ MINDLab, Center of Functionally Integrative Neuroscience, Aarhus University, Aarhus, Denmark \\ ${ }^{5}$ Institute of Behavioural Sciences, University of Helsinki, Helsinki, Finland
}

\section{Edited by:}

Marc Schönwiesner, University of Montreal, Canada

\section{Reviewed by:}

Marc Schönwiesner, University of Montreal, Canada

Bjørn Petersen, Royal Academy of

Music, Aarhus, Denmark

\section{*Correspondence:}

Ritva Torppa, Cognitive Brain

Research Unit, Institute of

Behavioural Sciences, University of

Helsinki, PO Box 9 ,

Siltavuorenpenger $1 B$,

Helsinki FIN-00014, Finland

e-mail: ritva.torppa@helsinki.fi
Informal music activities such as singing may lead to augmented auditory perception and attention. In order to study the accuracy and development of music-related sound change detection in children with cochlear implants $(\mathrm{Cls})$ and normal hearing $(\mathrm{NH})$ aged 4-13 years, we recorded their auditory event-related potentials twice (at T1 and T2, 14-17 months apart). We compared their MMN (preattentive discrimination) and P3a (attention toward salient sounds) to changes in piano tone pitch, timbre, duration, and gaps. Of particular interest was to determine whether singing can facilitate auditory perception and attention of $\mathrm{Cl}$ children. It was found that, compared to the $\mathrm{NH}$ group, the $\mathrm{Cl}$ group had smaller and later timbre P3a and later pitch P3a, implying degraded discrimination and attention shift. Duration MMN became larger from T1 to T2 only in the NH group. The development of response patterns for duration and gap changes were not similar in the $\mathrm{Cl}$ and $\mathrm{NH}$ groups. Importantly, $\mathrm{Cl}$ singers had enhanced or rapidly developing P3a or P3a-like responses over all change types. In contrast, $\mathrm{Cl}$ non-singers had rapidly enlarging pitch MMN without enlargement of P3a, and their timbre P3a became smaller and later over time. These novel results show interplay between MMN, P3a, brain development, cochlear implantation, and singing. They imply an augmented development of neural networks for attention and more accurate neural discrimination associated with singing. In future studies, differential development of P3a between $\mathrm{Cl}$ and $\mathrm{NH}$ children should be taken into account in comparisons of these groups. Moreover, further studies are needed to assess whether singing enhances auditory perception and attention of children with $\mathrm{Cls}$.

Keywords: ERPs (event-related potentials), development, attention, auditory memory, neuroplasticity, singing

\section{INTRODUCTION}

Music gives us pleasure and rewards us (Zatorre and Salimpoor, 2013). It helps us in regulation of emotions (Saarikallio, 2010) and even aids in maintaining the healthy functioning of memory and other cognitive functions in old age (Särkämö et al., 2014). Music listening could also be very important for severely hearingimpaired people using cochlear implants (CIs). Unfortunately, perception of music is difficult for CI-mediated listening (Limb and Roy, 2014). The CI substitutes for the sound reception and analysis functions of the inner ear by directly stimulating the fibers of the auditory nerve via an electrode system. The current CI processing strategies are based mostly on extraction and accurate representation of temporal envelopes of sounds (McDermott, 2004), while the spectral information provided to the auditory system is limited and fine structure of sounds is largely lost (Moore, 2003). Further, the dynamic range of electric hearing is highly limited (Moore, 2003; Limb and Roy, 2014). In consequence, the CI recipients have deficits in the ability to perceive many auditory cues important for music perception, like pitch and melody (adults: Drennan and Rubinstein, 2008; children:
Hsiao and Gfeller, 2012), timbre (Gfeller et al., 1998; Galvin et al., 2009; Timm et al., 2012) and loudness (Limb and Roy, 2014). However, their rhythm perception is close to normal (Drennan and Rubinstein, 2008; Limb and Roy, 2014).

The music perception of children with CIs (CI children) is affected not only by the degraded auditory input from the CI, but also by the period of deafness (Moore and Linthicum, 2007) and by cognitive factors (Rocca, 2012; Rochette et al., 2014) where the CI children show large variability (Kronenberger et al., 2014). Deafness during the first years of life may harm the development of attentional functions (Houston et al., 2003; Fagan and Pisoni, 2009) and their neural determinants (Moore and Linthicum, 2007). There is, however, almost a complete lack of studies on CI children's auditory attention even though it is important for their auditory learning (Houston and Bergeson, 2014) and for perception of degraded speech (Wild et al., 2012). Therefore, we investigate how effectively the CI children shift their attention toward changes in the auditory environment. Shifting of attention brings potentially important information into focus, allowing re-evaluation of the entire situation (Horváth et al., 2008). 
Longitudinal studies in normal-hearing $(\mathrm{NH})$ adults and children imply a causal link from musical training and hobbies to changes in brain structure and function (plasticity) (Herholz and Zatorre, 2012; Putkinen et al., 2013a, for a review), processing of sound features important for music (Moreno et al., 2009; Chobert et al., 2012) and auditory attention (Fujioka et al., 2006). It has been shown that formal musical training can facilitate the perception of pitch, melodic contour, musical timbre, and general music perception of CI users (Galvin et al., 2009; Yucel et al., 2009; Chen et al., 2010; Hsiao and Gfeller, 2012; Petersen et al., 2012; Limb and Roy, 2014) and it has been suggested that auditory attention of CI children can be facilitated with musical education (Rochette et al., 2014). In addition, informal musical activities at home, including singing, can facilitate the sound discrimination and attention of children (Putkinen et al., 2013a,b). Singing is an essential aspect in the development of musicality and has been recommended to be used in the rehabilitation of music perception of CI children (Rocca, 2012). Importantly, singing also captures and maintains the attention of young normal-hearing children (Nakata and Trehub, 2004) as well as CI children and is therefore effective in their speech therapy sessions (Ronkainen, 2011). Production of songs might play a special role for auditory attention because during singing the child not only hears but also feels the sounds in his/her articulatory apparatus, and multisensory stimuli recruit the attention of the young child (Bahrick and Lickliter, 2000). However, more research is needed on the role of informal singing in CI children's musical and attention development.

Here, we study how the CI children build up their music perception using singing. For our research purposes, we use event related potential (ERP) recordings. Mismatch negativity ERP response (MMN; Näätänen et al., 1978) is a cortical response to a moderate violation of regularity in the auditory scene (Wetzel et al., 2006). In NH listeners MMN has been shown to become larger in amplitude with more accurate perception and with effective discrimination training (Kujala et al., 2007). In the adult CI users, MMN becomes larger with more accurate discrimination of musically relevant sound changes such as pitch, rhythm and timbre (Timm et al., 2014) and increased time of CI use (Lonka et al., 2013). MMN latencies, in turn, are shortened by increasing physical difference between the standard and deviant tone (Kujala et al., 2007). MMN can be followed by a P3a response, which reflects an involuntary attention switch toward a salient sound change in the auditory environment (Escera et al., 1998; Wetzel et al., 2006). Like MMN, P3a for deviant tones becomes larger with increasing physical difference between the deviant and standard stimulus (Winkler et al., 1998; Wetzel et al., 2006) and with effective auditory training (Uther et al., 2006). Further, P3a is larger and earlier in CI children with better speech recognition (Kileny et al., 1997). P3a is thought to reflect evaluative discrimination related to the activation of an attentional switch mechanism (Friedman et al., 2001; Horváth et al., 2008). This is in contrast to the pre-attentive detection of deviant events reflected by the MMN (Tremblay et al., 1998; Friedman et al., 2001; van Zuijen et al., 2006). It seems that the P3a is elicited when the sound change is significant and intrusive for the listener (Horváth et al., 2008).
We measured the MMN and P3a responses to changes in sound pitch, timbre, duration, gap, and intensity in a passive multi-feature paradigm (MFP) (Näätänen et al., 2004; Torppa et al., 2012) using instrumental sounds. We chose to use MFP since it provides a comprehensive view of the basic auditory processing during a single recording in a shorter period of time than with traditional oddball paradigms. This is highly beneficial in child measurements (Lovio et al., 2009), which was important for the present study where the participants were aged from four to thirteen years. There is almost a complete lack of studies on the neural encoding of the sound feature changes in musical instrument stimuli in deaf-born, early-implanted CI children (however, see Torppa et al., 2012) and nothing is known about the development of MMN and P3a to musical instrument sounds in children, within the time period we are concentrating on (1417 months). The CI children participating the present study, however, were implanted so early (at or before age of 3 years, 1 month) that they had the possibility to develop many auditory functions similar to NH children (Kral and Sharma, 2012). Because typically the amplitude of ERP responses increases and latency decreases as a function of auditory experience with CIs (P1 latency: Kral and Sharma, 2012; MMN amplitude: Ponton et al., 2000; Lonka et al., 2013; P3a amplitude: Kelly et al., 2005), we expect that the MMN and $\mathrm{P} 3 \mathrm{a}$ of the present participants with CIs would develop more during our follow-up than those of $\mathrm{NH}$ children.

We tested two hypotheses based on the introduction above: (I) The CI children have smaller and/or later MMN and P3a than $\mathrm{NH}$ children for the changes in timbre and pitch (while not for the rhythm-related changes i.e., gaps and duration changes): the differences between groups become smaller over time. (II) The MMN and P3a is/becomes larger and/or earlier in CI children who sing regularly at home compared to other CI children. To follow and compare development between groups, the experiment was performed twice. To test Hypothesis II, we divided the CI children into two groups based on the regularity and amount of their long-term singing at home: "CI singers" and "CI non-singers."

\section{MATERIALS AND METHODS PARTICIPANTS}

The CI participants of this study (CI group) were 21 (9 male, 12 female) unilaterally implanted, congenitally deaf, Finnishspeaking children aged from 4 to 13 years during the course of this study (Table 1). They were chosen from a total of 30 children with a CI. Inclusion criteria were switch-on of the CI prior to three years one month, no re-implantation between the two measurements, more than 6 CI channels in use, and the absence of any diagnosed additional developmental or linguistic problems. All CI children had been using their implants continuously for at least 30 months prior to first measurement (T1) and at least 46 months prior to the second measurement (T2), had full insertion of the electrode and attended mainstream school or day care. Seventeen CI children used Nucleus and four used Medel devices (Table 1). According to the clinical recordings, their hearing thresholds in the unimplanted ear were so high that they could not benefit from residual hearing 
Table 1 | Characteristics of the participants.

\begin{tabular}{|c|c|c|c|c|c|c|c|c|c|}
\hline$I^{a}$ & Age at T1 & Hand $^{b}$ & Music $^{c}$ & $S^{d}$ & Etiologye & $\begin{array}{l}\text { Age at } \\
\text { switch-on of } \\
\mathrm{Cl} \text { (months) }\end{array}$ & $\begin{array}{l}\text { Cl use prior } \\
\text { T1 (months) }\end{array}$ & $\begin{array}{l}\text { Cl processor } \\
\text { type }^{f}\end{array}$ & $\begin{array}{l}\text { Pure tone } \\
\text { thresholds using } \mathrm{Cl} \\
\text { (dB HL) }\end{array}$ \\
\hline Cls 03 & $9 y 2 m$ & $\mathrm{R}$ & 12 (betw) & $\mathrm{R}$ & U & 32 & 77 & MT & 35/28/-/- \\
\hline Cls 04 & $7 y 10 m$ & $\mathrm{R}$ & 24(betw) & $\mathrm{R}$ & $U$ & 25 & 69 & MT & $25 / 23 / 25 / 25$ \\
\hline Cls 14 & $4 y 4 m$ & $\mathrm{R}$ & 0 (betw) & $\mathrm{R}$ & $U$ & 18 & 34 & NF & $15 / 17 / 20 / 30$ \\
\hline Cls 15 & $5 y 1 m$ & $\mathrm{R}$ & 0 & $\mathrm{R}$ & $\mathrm{C}$ & 17 & 44 & NE & $45 / 43 / 40 / 40$ \\
\hline Clns 16 & $7 y 2 m$ & $\mathrm{R}$ & 0 & $\mathrm{R}$ & $\mathrm{C}$ & 25 & 61 & NF & $25 / 20 / 35 / 35$ \\
\hline Clns 17 & $9 y 4 m$ & $\mathrm{~L}$ & 0 & $\mathrm{R}$ & U & 19 & 93 & NF & $30 / 25 / 25 / 40$ \\
\hline CIns 18 & $12 \mathrm{y} 1 \mathrm{~m}$ & $\mathrm{R}$ & 0 & $\mathrm{R}$ & $U$ & 27 & 118 & NF & 25/15/30/45 \\
\hline Cls 22 & $7 y 1 m$ & $\mathrm{R}$ & 0 & $\mathrm{R}$ & U & 21 & 48 & NE & $35 / 28 / 35 / 35$ \\
\hline CIns 23 & $7 y 10 m$ & $L$ & 0 & $\mathrm{R}$ & U & 18 & 76 & MT & $30 / 25 / 30 / 30$ \\
\hline CIns 24 & $4 y 2 m$ & $\mathrm{R}$ & 23(betw) & $\mathrm{R}$ & C & 14 & 36 & NF & $20 / 20 / 25 / 40$ \\
\hline Cls 26 & $4 y 2 m$ & $\mathrm{R}$ & 23(betw) & $\mathrm{R}$ & $\mathrm{C}$ & 20 & 30 & $N F$ & 20/23/30/- \\
\hline Clns 27 & $4 y 2 m$ & $\mathrm{R}$ & 0 & $\mathrm{R}$ & $\mathrm{C}$ & 13 & 37 & NF & 25/30/30/- \\
\hline Cls 28 & $6 y 2 m$ & $\mathrm{R}$ & 24 & $\mathrm{R}$ & U & 22 & 52 & NF & $10 / 10 / 10 / 55$ \\
\hline CIns 29 & $8 y 7 m$ & $\mathrm{R}$ & 0 & L & $\mathrm{C}$ & 37 & 66 & NF & $25 / 28 / 30 / 25$ \\
\hline Cls 30 & $6 y 7 m$ & $\mathrm{R}$ & 0 & $\mathrm{R}$ & $\mathrm{C}$ & 25 & 54 & NF & 40/28/30/- \\
\hline $\begin{array}{l}\mathrm{NCl}=21 \\
\mathrm{~N} \mathrm{Cls}=12 \\
\mathrm{NClns}=9\end{array}$ & $\begin{array}{l}\text { Mean = } \\
6 y 7 m\end{array}$ & $N R+L=18+3$ & $\begin{array}{l}N \text { attend: } \\
\text { before }=7 \\
\text { betw }=8\end{array}$ & $\mathrm{~N} \mathrm{R}+\mathrm{L}=19+2$ & $\begin{array}{l}\mathrm{NU}=12 \\
\mathrm{NC}=9\end{array}$ & Mean $=21.7$ & Mean $=58.1$ & $\begin{array}{l}\text { N NF }=13 \\
\text { N NE }=4 \\
\text { N MO }=1 \\
\text { N MT }=3\end{array}$ & $\begin{array}{l}\text { Mean Cls } \\
\text { 28/26/29/38 } \\
\text { Mean CIns } \\
\text { 26/25/31/36 }\end{array}$ \\
\hline $\mathrm{NH} 06$ & $5 y 8 m$ & $\mathrm{R}$ & 0 (betw) & & & & & & \\
\hline $\mathrm{NH} 07$ & $6 y 9 m$ & $\mathrm{R}$ & 0 & & & & & & \\
\hline $\mathrm{NH} 08$ & $5 y 7 m$ & $\mathrm{R}$ & 0 (betw) & & & & & & \\
\hline $\mathrm{NH} 09$ & $4 y 6 m$ & L & 42 (betw) & & & & & & \\
\hline $\mathrm{NH} 10$ & $4 y 0 m$ & $\mathrm{R}$ & 0 (betw) & & & & & & \\
\hline $\mathrm{NH} 11$ & $5 y 6 m$ & $\mathrm{R}$ & 0 & & & & & & \\
\hline $\mathrm{NH} 13$ & $5 y 0 m$ & $\mathrm{R}$ & 35 (betw) & & & & & & \\
\hline $\mathrm{NH} 14$ & $4 y 6 m$ & $\mathrm{R}$ & 15(betw) & & & & & & \\
\hline $\mathrm{NH} 15$ & $12 y 0 m$ & $\mathrm{R}$ & 0 & & & & & & \\
\hline $\mathrm{NH} 16$ & $8 y 5 m$ & $\mathrm{R}$ & 0 & & & & & & \\
\hline $\mathrm{NH} 17$ & $9 y 8 m$ & $\mathrm{R}$ & 0 & & & & & & \\
\hline $\mathrm{NH} 18$ & $6 y 9 m$ & $\mathrm{R}$ & 0 & & & & & & \\
\hline NH 19 & $7 y 0 m$ & $\mathrm{R}$ & 0 & & & & & & \\
\hline $\mathrm{NH} 20$ & $4 y 6 m$ & $\mathrm{R}$ & 12 & & & & & & \\
\hline $\mathrm{NH} 21$ & $6 y 5 m$ & $\mathrm{R}$ & 15 & & & & & & \\
\hline NH 22 & $6 y 11 \mathrm{~m}$ & $\mathrm{R}$ & 0 (betw) & & & & & & \\
\hline
\end{tabular}


Table 1 | Continued

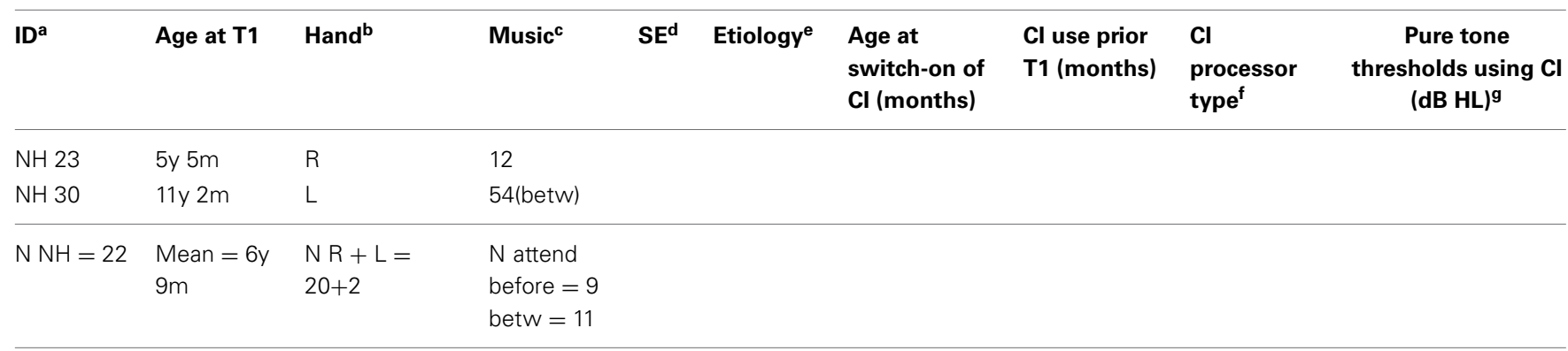

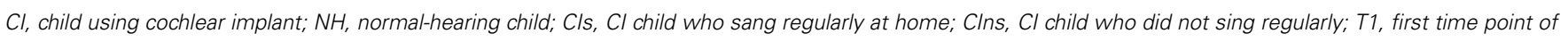
measurements; T2, second time point of measurements; $N$, number.

* Included in analyses but data only from T2.

a Identification number.

${ }^{b}$ Hand, handedness.

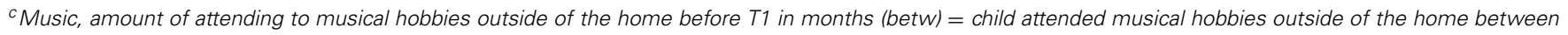
measurements (dancing excluded).

${ }^{d} S E$, stimulated ear.

e $U$, unknown, C, Connexin 26.

${ }^{f} \mathrm{NF}$, Nucleus Freedom, implant type CIC4 (coding strategy: ACE).

$N E$, Nucleus ESPrit 3G, implant type CIC3 (coding strategy: ACE).

MT, Medel Tempo + (coding strategy: CIS).

MO, Medel Opus 2 (coding strategy: CIS).

${ }^{g}$ For $4000 \mathrm{~Hz} /$ for mean of 500,1000 , and $2000 \mathrm{~Hz} /$ for $250 \mathrm{~Hz} /$ for $125 \mathrm{~Hz}$.

in the present ERP measurements. They all also participated in the study by Torppa et al. (2012) and Torppa et al. (2014), as did the 22 normal-hearing children who served as a control group (NH group).

The $22 \mathrm{NH}$ children (11 male, 11 female) were siblings of the participating CI children or were invited for this study from the neighborhood of the first author, from local musical play schools, and from another ongoing study at the University of Helsinki. The $\mathrm{NH}$ group was matched to the CI group in group level as accurately as possible by age, gender, handedness as well as social and musical background, the latter as assessed by attendance in musical playschool or related musical activities (see Table 1). None of the NH children had any diagnosed developmental or linguistic problems. Their hearing was found to be normal in regular check-ups at child welfare clinics.

Parents of the participants gave a written informed consent and the participants gave their consent verbally. The study was carried out in accordance with the Declaration of Helsinki and all procedures were approved by the ethical committees of the participating hospitals.

\section{Division of $\mathrm{Cl}$ group into $\mathrm{Cl}$ singers and $\mathrm{Cl}$ non-singers}

We made the division with similar principles as in the previous child studies comparing children with and without musical training (schools with emphasis on music, Putkinen et al., 2014; private instrument lessons, Strait et al., 2012; see Margulis, 2008, for different criteria for adult musicians vs. non-musicians). Therefore, we chose the CI singers on the basis of the regularity of their musical activity (singing) in the home and the time they had sung before the study began. For the division, information about the children's musical and other hobbies as well as musical activities at home, school and day care was collected with questionnaires addressed to parents and personnel at schools or day care. As a measure to divide the CI children into those who sing regularly at home and those who do not, the parents were asked to account for their children's singing activities including those with parents, siblings or alone: "Has your child sung at home? Every week_every other week_occasionally_ not at all_ if weekly, how many times in a week" at T1: during the previous year, at T2: between the measurements. According to their answers, 12 CI children sang weekly at home one year before the study began and between T1 and T2 and these are hence called "CI singers." They sang at home on average five times per week before T1 at and 4 times per week between T1 and T2. Nine CI children sang less than weekly or not at all and are called "CI nonsingers." They sang at home on average less often than every other week (=occasionally) before $\mathrm{T} 1$ and between $\mathrm{T} 1$ and T2. In addition, all CI children had at school or day care music lessons with an emphasis on singing.

To ensure that the parents were able to identify singing of the CI children as different from speech, we recorded their singing ("Twinkle twinkle little star") when they came to the measurements at T2. The parents and children were told about the task beforehand. Nineteen out of 21 CI children completed the task. The rhythm, melody and words (lyrics) they sang were scored blindly, i.e., without knowing whether the child was CI singer or not, by a teacher of singing. As a result, $15 \mathrm{CI}$ children could sing the lyrics correctly and 4 of them partially correctly. Nine of them could sing the rhythm correctly and 6 partially correctly. One CI child sang the melody almost correctly, 5 could vary their pitch partially correctly, 7 varied the pitch to some extent: 6 sang without variation in pitch, however, all of them could produce the rhythm and lyrics correctly or partially. It was concluded that their singing was recognizable and different 
from general speech. The interviews of parents also supported this conclusion.

The comparisons between CI singers and CI non-singers showed that the accuracy of production of lyrics, melody, and rhythm was better in CI singers than in CI non-singers. Agecontrolled ANOVA confirmed that the CI singers were significantly better in production of rhythm $\left[F_{(1,18)}=7.83, p=0.013\right]$ and in the overall accuracy of singing (the mean of production of lyrics, melody and rhythm) $\left[F_{(1,18)}=5.28, p=0.035\right]$ than CI non-singers. In contrast, statistical analyses confirmed that these CI singing groups did not differ from each other in the other home-related musical background (including musical instrument playing), amount of musical activities at day care or schools, in musical hobbies, or in the aspects related to their hearing or CI devices, age, gender, socioeconomic background, or etiology (see Supplement 1).

\section{STIMULI AND PROCEDURE}

The stimuli and procedure were the same as in Torppa et al. (2012). Piano, harpsichord (cembalo), violin, and cymbal sounds selected from McGill University Master Samples DVD were cut from the beginning of the original samples to the desired duration and normalized in intensity with Adobe Audition 2.0 (Adobe Systems Inc., San Jose, USA). The standard was a piano tone at $295 \mathrm{~Hz}$, duration $200 \mathrm{~ms}$ including a $20 \mathrm{~ms}$ fall time. The deviant tones differed from the standards at three different levels of fundamental frequency (pitch), all harmonics changing from $295 \mathrm{~Hz}$ standard to 312,351 , and $441 \mathrm{~Hz}$ corresponding to 1,3 , and 7 semitones respectively, timbre (change from standard piano tone to cembalo, violin, and cymbal tone) (see Figure 1), duration (shortening of $200 \mathrm{~ms}$ standard to $175 \mathrm{~ms}, 100$ and $50 \mathrm{~ms}$ ), intensity (increments and decrements, $\pm 3, \pm 6$, and $\pm 9 \mathrm{~dB}$ ) or by the presence of a silent gap in the middle of the tone $(5,40$, and $100 \mathrm{~ms}$ gaps). The deviant tones were equivalent to the standard in all other features, except in the $50 \mathrm{~ms}$ deviant, where the fall time was $10 \mathrm{~ms}$, and for the timbre changes, which contained changes in temporal intensity envelope, spectral envelope, and periodicity. In the stimulus sequence standard and deviant tones alternated. The SOA was kept at $480 \mathrm{~ms}$. The presentation order of the changes was randomized throughout the experiment. The probability of the standard tone was 0.5 and the probability of each change was 0.028 . There were 2250 standard tones and 125 deviants. The total recording time of the experiment was $36 \mathrm{~min}$.

All stimuli were presented in an acoustically insulated and dampened room through 2 loudspeakers (OWI-202; OWI Inc. CA., USA) placed at a $45^{\circ}$ angle to each side of the subject, approximately $1 \mathrm{~m}$ in distance from the subject's ear. The stimuli were presented at a fixed and comfortable level, which was $60 \mathrm{~dB}$ SPL for NH group and $70 \mathrm{~dB}$ SPL for CI group (in exception to the intensity changes). For one CI child the sound level had to be lowered to $65 \mathrm{~dB}$ SPL at T1. The sounds were presented using the everyday settings of the CI. Program, and volume and sensitivity level were adjusted to the clinically recommended values. During the experiment, subjects watched a silent video.

\section{EEG RECORDING, DATA PREPROCESSING AND DATA ANALYSIS}

EEG recordings were conducted from a 64-channel electrode cap using Biosemi ActiveTwo amplifier and active electrodes (Biosemi B.V., Netherlands) (sampling rate of $512 \mathrm{~Hz}$, band-pass filtering of DC-102.4 Hz). The data were referenced on-line to the CMS electrode according to the basic setup of the Biosemi measurement device and off-line data was re-referenced to the electrode

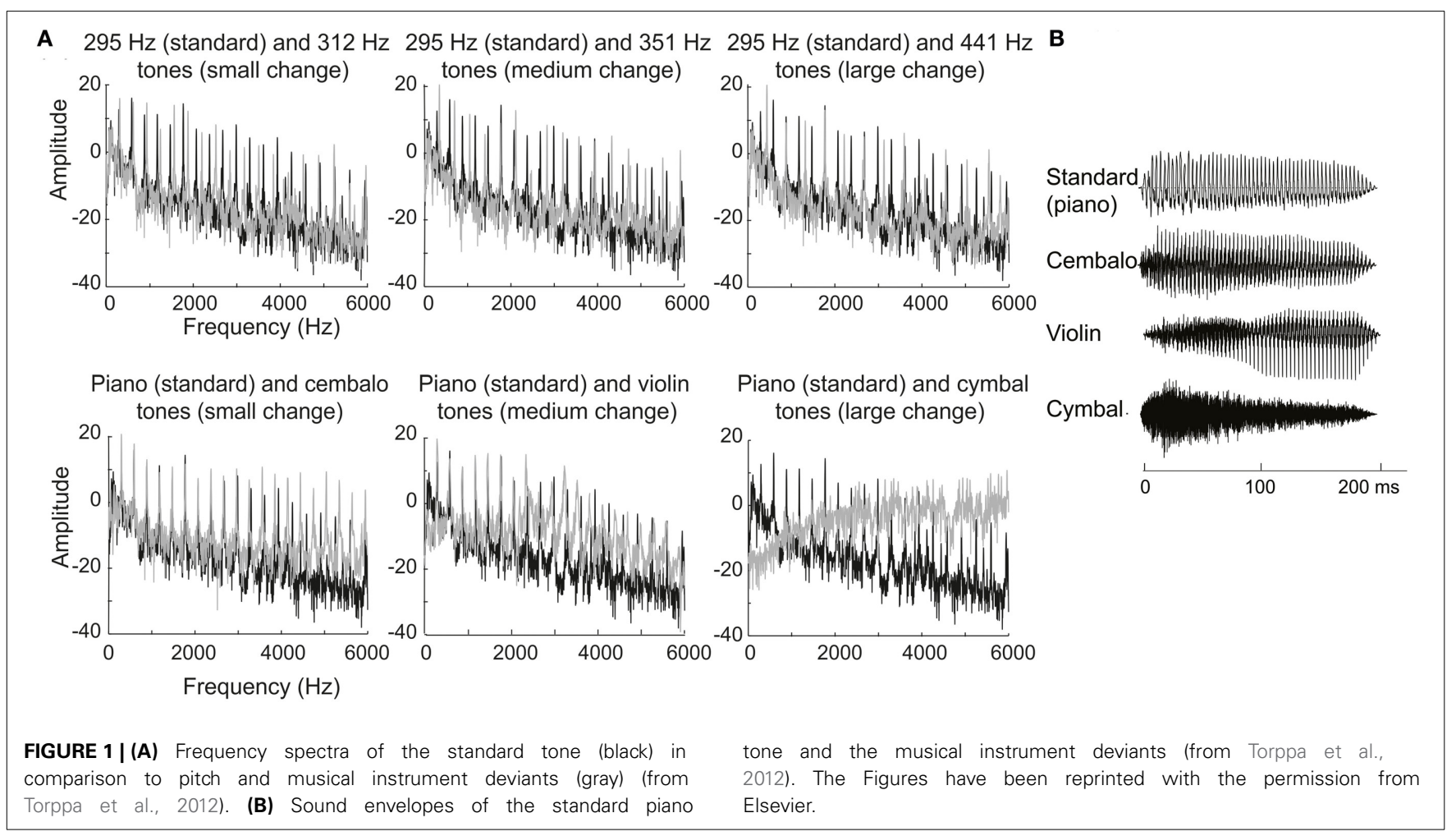


placed at the nose tip. Additional electrodes were placed at the left and right mastoid and to record eye movements and blinks. EEG data were analyzed using EEGLAB 8 (Delorme and Makeig, 2004). Imported data were downsampled at $256 \mathrm{~Hz}$, and highpass filtered above $0.5 \mathrm{~Hz}$. Because of the location of the CI device, some channels could not be used; data from these electrodes were interpolated. The analysis epoch was $550 \mathrm{~ms}$ long, starting $100 \mathrm{~ms}$ before the onset of the tones. The baseline level of the epochs was set to be zero during the $100 \mathrm{~ms}$ before the tone onsets.

Independent component analysis (ICA) with the Fastica algorithm was applied to remove ocular and muscle artifacts in both CI and NH groups (Makeig et al., 2004). In addition, ICA was used in the CI group to reduce the CI related artifact. Several previous studies indicate that ICA is the best available method for this (Gilley et al., 2006; Sandmann et al., 2010: for the CI artifact component, see Torppa et al., 2012). Before ICA, data dimensionality was narrowed down by the amount of interpolated channels, and automatic epoch rejection at a threshold between \pm 300 and $\pm 400 \mu \mathrm{V}$ was performed. The rejection thresholds were individually adjusted to preserve at least $85 \%$ of original epochs for effective statistical analysis. After ICA, the epoch voltage rejection was done again with a threshold of $\pm 150 \mu \mathrm{V}$. Further, the proportion of remaining epochs after voltage rejection was analyzed for each individual subject. The criteria of $75 \%$ (95) remaining epochs for each deviant was used. One participant did not reach the criteria at $\mathrm{T} 1$ and so her data was excluded from the analyses. All participants reached the criteria at T2 and thus the data from all children at T2 was used in further analyses. The mean percentage of acceptance of epochs at T1 was $94 \%$ in the CI group (119 deviants, 2348 standards) and 93\% in the $\mathrm{NH}$ group (116 deviants, 2330 standards), and at $\mathrm{T} 2$ was $93 \%$ in the CI group (116 deviants, 2330 standards) and 95\% in the NH group (119 deviants, 2348 standards). To increase the signal to noise ratio (especially for noise sensitive latency measures), and to make the analyses more comparable between groups, F3, $\mathrm{Fz}, \mathrm{F} 4, \mathrm{C} 3, \mathrm{Cz}$, and C4 channels were averaged to form a ROI (region of interest) channel. This procedure was used in all ERP analyses.

Calculating the median instead of average of ERP signals is optimal in cases where the data are of high quality in general, but some overlapping noise seen as extreme values is expected, when studying young children (Yabe et al., 1993). Hence we chose to use this median method in the present study (for differences between median and averaged signals, see Supplements 2, 3). First, the trials of each individual were grouped by stimulus type. After this, the median value of the signal amplitude values of one sample point was taken as representative of that sample point. Thus, the resulting curve from an individual consists of the samples having the median amplitude over the accepted trials. Further, the data were offline-filtered with a $25 \mathrm{~Hz}$ low-pass filter and the baseline level of the epochs was set to be zero during the $50 \mathrm{~ms}$ period before the tone onsets.

MMN was identified as the local minimum (most negative peak) of the subtraction waveform within the time window 90-250 ms after change onset. P3a was identified as the local maximum of the subtraction waveform within the time window 145-300 ms after change onset. Gap MMN and P3a was defined in relation to offset of the gap due to the clear tendency of the CI group to have MMN only for the offset of $100 \mathrm{~ms}$ gap. The mean amplitudes were calculated using a $30 \mathrm{~ms}$ time window surrounding the peak latency. The individual peak latencies were calculated from the ROI-signal in a time window determined in relation to the onset of the stimulus change. For gap changes, the time window was determined in relation to the offset of the gap, and for duration changes, in relation to offset of the deviant tone. Based on visual inspection of the data, the window was set at $85-250 \mathrm{~ms}$ for pitch and timbre MMN, at 100-250 ms for duration and gap MMN and at 145-400 ms for P3a. The onset of the timbre and pitch MMN latency window was early, since the earliest individual MMN for which we could confirm polarity change at mastoid electrodes compared to $\mathrm{Fz}$ was found for timbre changes at $86 \mathrm{~ms}$ and for pitch changes at $89 \mathrm{~ms}$. In addition, we analyzed the amplitudes and latencies of the intensity decrement and increment $\mathrm{MMN}$ and $\mathrm{P} 3 \mathrm{a}$ responses (analyses and results are provided in Supplement 4).

ERP response amplitudes and latencies were subjected to onesample, two-tailed $t$-tests in order to examine whether they significantly differed from zero in the $\mathrm{CI}$ and $\mathrm{NH}$ group. In order to compare MMN and P3a between $\mathrm{CI}$ and $\mathrm{NH}$ groups or between $\mathrm{CI}$ singers and CI non-singers, we took into analysis the responses with following principles. The response for the specific deviant type was included in the analyses, if the MMN/P3a was significant at T1 and/or T2 for the both tested child groups. Cases, where the significant response was not found either at T1 or T2, were still included in the analyses, because the standard deviation of the responses were similar in both measurement points (see Table 2).

\section{STATISTICAL ANALYSES}

The analyses for testing the hypotheses were conducted with Linear Mixed Model (LMM; Singer and Wilett, 2003; West, 2009). This multilevel statistical procedure enables studying individual change over time to fit a variety of advanced regression models to longitudinal data sets. LMM was used because (a) it takes into account the missing values, which makes it possible to utilize data from all participants even though the data at one of the two time points are missing (as mentioned before, we had to exclude data from one CI participant at T1), (b) LMM allows one to combine measurements from the two time points in a single analysis, which allowed us to compare the development of the ERP indices of auditory perception and attention over time in $\mathrm{CI}$ and $\mathrm{NH}$ participants.

Two LM regression analysis models were created to test the hypotheses I and II. According to the hypotheses, the first LMM included factor CI vs. NH group (group) and the second LMM included factor CI singers vs. CI non-singers (CI singing group). In both LMMs, the amplitude or latency values from recording times T1 and T2 were included (factor time). Accordingly, the interactions of time with group or CI singing group were tested to determine differences between child groups in development. In both LM models, amount of change was also a factor, and twoand three-way interactions of that with group or CI singing group and time were tested. Two-way interactions were tested to find out whether there would be differences between aforementioned 
Table 2 | The MMN and P3a mean amplitudes and latencies.

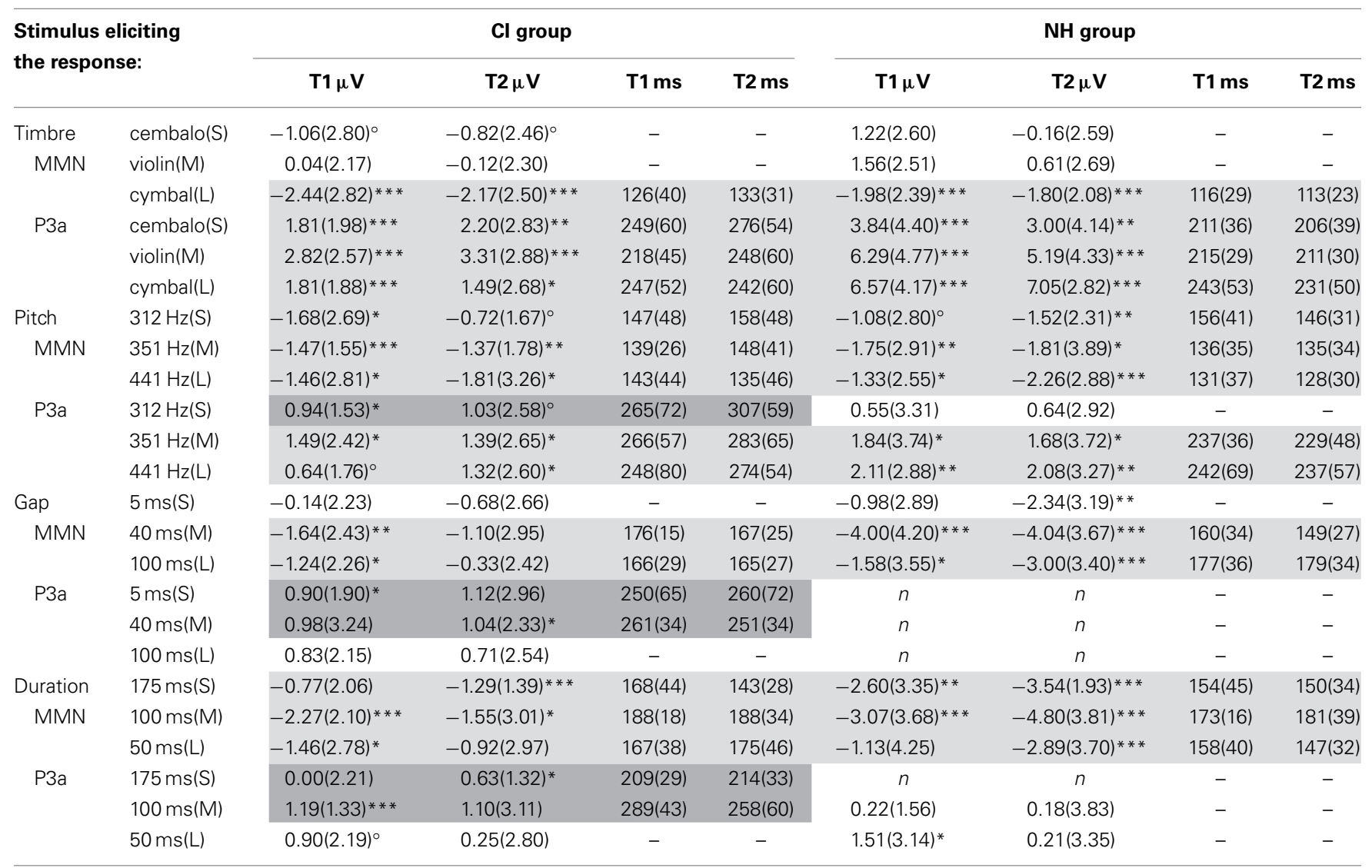

For both $\mathrm{Cl}$ and $\mathrm{NH}$ groups, the mean MMN and P3a amplitude (standard deviation in parenthesis), followed by the significance of the response ${ }^{\circ} \mathrm{p}<0.1,{ }^{*} p<$ $0.05,{ }^{*} p \leq 0.01,{ }^{* *} p<0.001$; two-tailed t-test against zero), are given at T1 and T2. These are followed by the mean of peak latencies (standard deviation in parenthesis). Light gray presents the amplitude and latency values included in statistical comparisons between $\mathrm{Cl}$ group and $\mathrm{NH}$ group as well as between $\mathrm{Cl}$ singers and $\mathrm{Cl}$ non-singers. Dark gray presents the values included only in statistical comparisons between $\mathrm{Cl}$ singers and $\mathrm{Cl}$ non-singers. $-=$ the individual latencies were not analyzed. $n=$ the responses were non-existent (wrong polarity in the time window of the response). Standard was $295 \mathrm{~Hz}, 200 \mathrm{~ms}, 70 \mathrm{db}$ (for $\mathrm{Cl}$ group)/60 $\mathrm{dB}$ (for $\mathrm{NH}$ group) piano tone. $S, M, L=$ small, medium and large amount of change.

groups only in some particular amount of change, and three-way interactions were tested to find out whether there would be differences in the development between any of the groups only in some particular amount of change. We included age as a covariate in all analyses to control for the large age range of the participants. Interactions with age were also tested because interaction of age with group, CI singing group or time would indicate that age could not be controlled. Only the significant interactions of age are reported in Tables 3, 4. All non-significant interactions were omitted from the final results reported in Tables 3, 4. Because the amounts of changes across the change types were not matched, the hypotheses had to be tested separately for each change type.

Importantly, in multilevel analyses, the covariance structure must always be taken into account. LMM procedure allows testing and usage of the best fitted covariance structure. Therefore, a statistical correction like Greenhouse-Geisser is not needed. We tested the covariance structures and selected the best fitting ones based on Akaike's and Bayesian information criteria (AIC and BIC) (Bryk and Raudenbush, 2002). AIC is a measure of the relative quality of a statistical model for a given set of data (Akaike,
1974), while BIC is a criterion for model selection among a finite set of models (Schwarz, 1978). The results are reported with the best fitting structure. The Bonferroni correction was used for all post-hoc tests within each model and for those tests, only the Bonferroni corrected $p$ values are given.

In Torppa et al. (2012), small or non-existent MMN preceding early P3a for changes in timbre was found. This suggested that the small MMN was consequence of the partial overlap of the P3a with the MMN, i.e., that the P3a was elicited early, at the latency where the MMN was assumed to be elicited. If in the present study the MMN preceding the P3a was unexpectedly small, we conducted partial correlation analysis (age controlled) between the amplitudes of the MMN, or the ERP-responses in the expected time line of the MMN, and the amplitudes of the following P3a. If the correlation was positive, the $\mathrm{MMN}$ became smaller with enlargement of the P3a, and the overlap was evident.

In general, the critical level for significance was set at 0.05 , and only the significant results related to the hypotheses are reported in the Results Section. All statistical analyses were made with SPSS 20 software. 

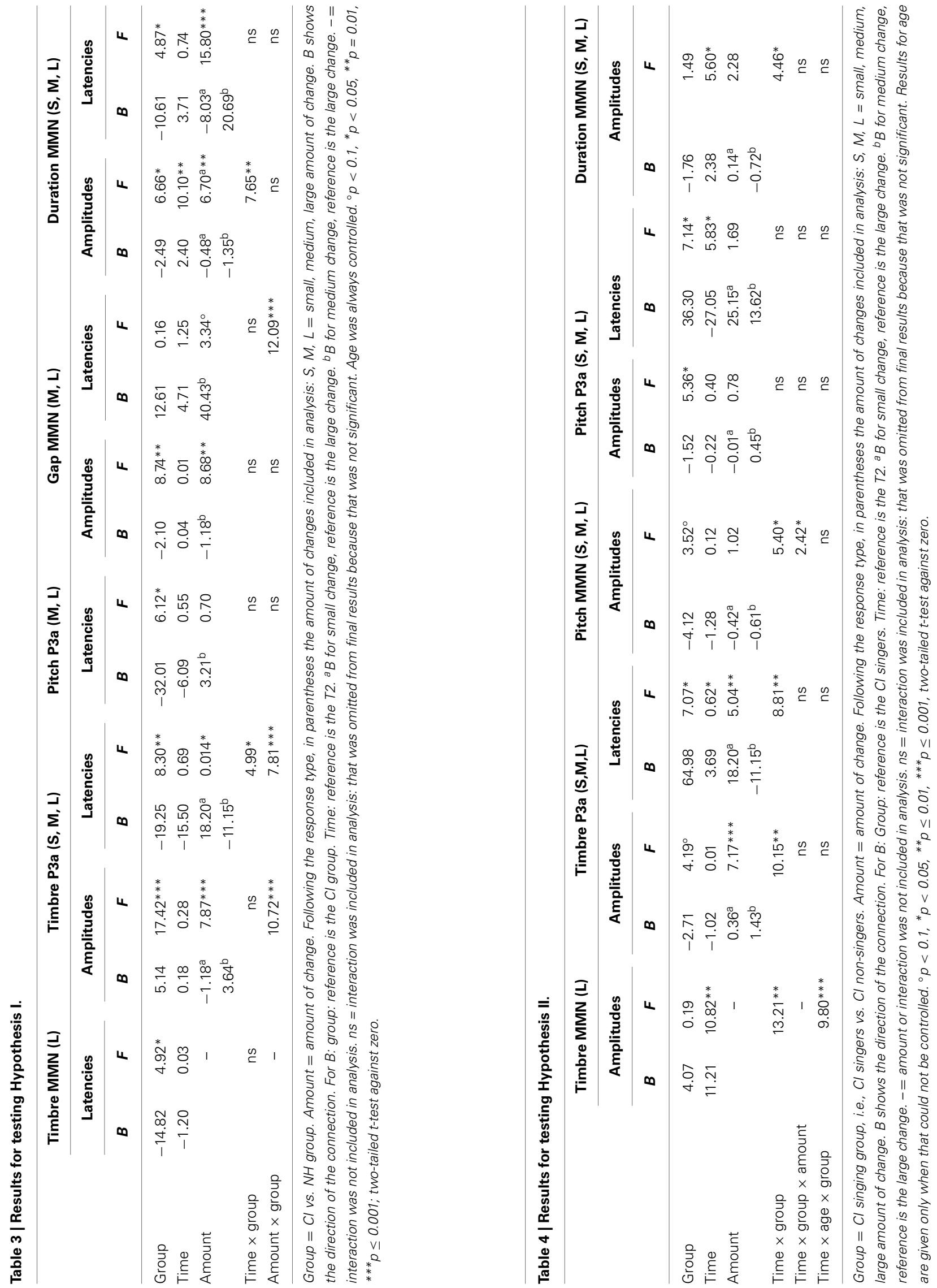


\section{RESULTS}

Table 2 shows the mean amplitudes and the latencies of the $\mathrm{MMN}$ and $\mathrm{P} 3 \mathrm{a}$ responses for the timbre, pitch, gap, and duration deviants for the $\mathrm{CI}$ and $\mathrm{NH}$ groups. Results related to intensity increments and decrements are provided in Supplement 4.

\section{TIMBRE AND PITCH RESPONSES WERE SMALL AND/OR LATE IN THE CI GROUP}

Timbre MMN latency was longer and timbre P3a was smaller and later in the CI group than in the $\mathrm{NH}$ group (main effects of group, Table 3; Figure 2A, for ERP waveforms, Supplement 5). Because there was a significant interaction of group and amount of change for both P3a amplitude and latency (Table 3), we conducted post-hoc tests. We found that the difference between the groups was significant for each amount of timbre change (medium change, i.e., from piano to violin: amplitude, $p=0.003$; large change, i.e., to cymbal, amplitude, $p<0.001$; small change, i.e., to cembalo, latency, $p<0.001$ ). In addition, timbre P3a latency became longer over time only in the CI group (interaction of group and time, Table 3; post-hoc test, $p=0.035$ ).

Moreover, in the $\mathrm{NH}$ group, the MMN was non-existent for the change from piano to cembalo and to violin (Figure 2A). This was also the case in the CI singers (Figures 3A, 4A). Therefore, we conducted the partial correlation analyses for all participants between the amplitudes the timbre MMN (at the latency it was assumed to be elicited) and the timbre P3a. We found that MMN and P3a amplitudes for the changes to cembalo and to violin correlated significantly (at T1, cembalo, $r_{p}=0.48, p=0.001$; violin, $r_{p}=0.65, p<0.001$; at T2 violin, $\left.r_{p}=0.49, p=0.001\right)$ : the MMN was smaller when the $\mathrm{P} 3 \mathrm{a}$ was larger. This correlation suggests a co-dependence and a possibly overlapping MMN and P3a (see Discussion).

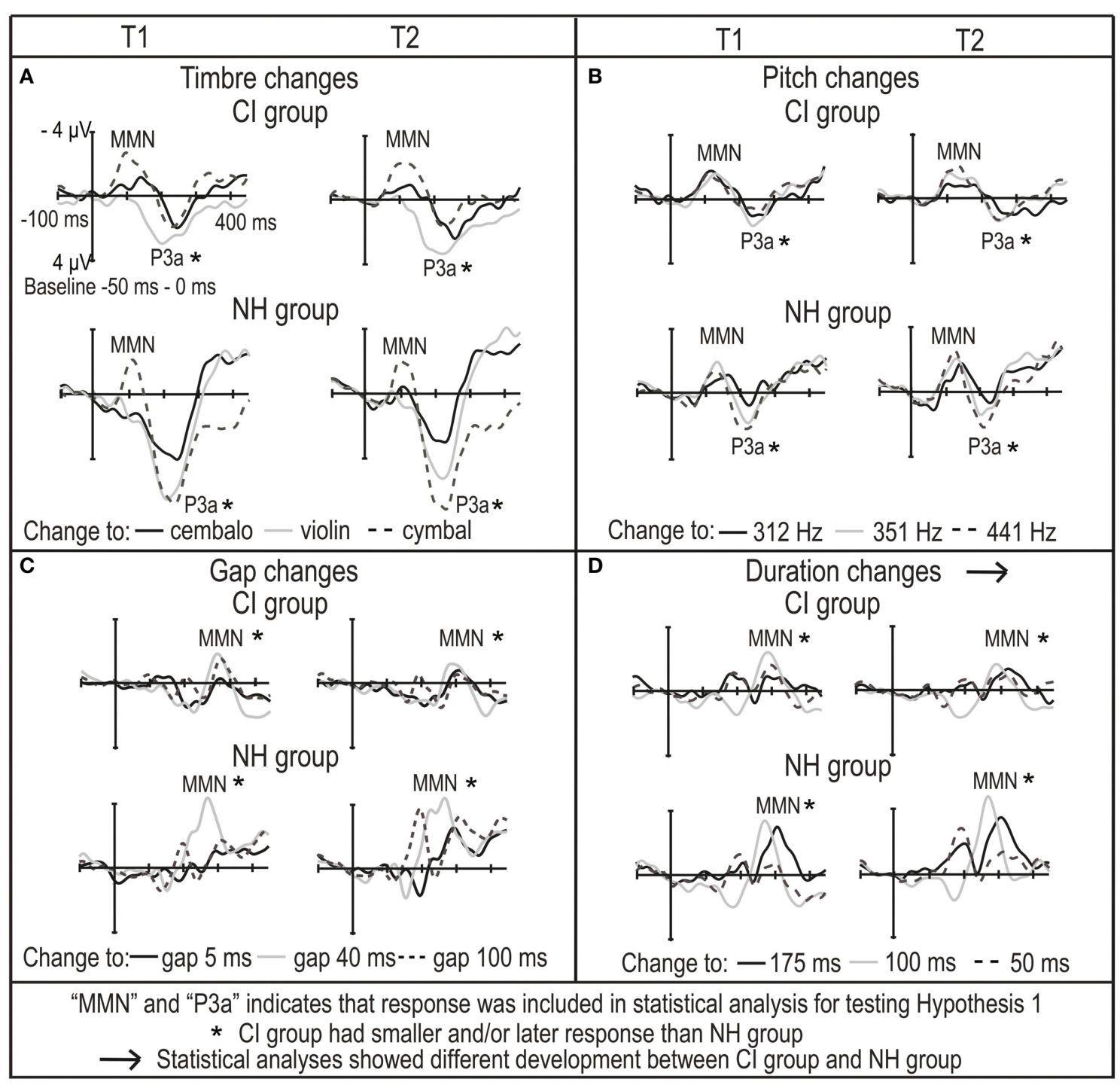

FIGURE 2 | The subtraction (deviant-standard) ROI waveforms averaged across $\mathrm{F} 3, \mathrm{Fz}, \mathrm{F} 4, \mathrm{C3}, \mathrm{Cz}$ and $\mathrm{C} 4$ electrodes for $\mathrm{Cl}$ and NH group for (A) timbre changes, (B) pitch changes, (C) gap changes and (D) duration changes. They are given for both time points of the measurements (T1 and T2 on the left and right in each panel, respectively). 
Pitch P3a latency was longer in the CI group than in the NH group (main effect of group, Table 3, Figure 2B). Gap MMN amplitude was smaller and latency for the medium gap longer in the CI group compared to the NH group (amplitude, main effect of group, Table 3; latency, interaction of amount of change and group, Table 3; post-hoc test, difference between CI and $\mathrm{NH}$ group for the medium change, $p=0.013$ ) (Figure 2C, Supplement 5). Importantly, gap P3a was elicited in the CI group only (Figure 2C). We also conducted partial correlation analyses between the amplitudes of the MMN and P3a in the CI group. They correlated significantly (small gap, at T1, $r_{p}=0.59, p=$ 0.008 , at T2, $r_{p}=0.67, p=0.001$; medium gap, at T1, $r_{p}=0.54$, $p=0.018$, at T2, $r_{p}=0.55, p=0.012$; large gap, at T2, $r_{p}=0.58$, $p=0.007)$. The MMN was smaller with the larger $\mathrm{P} 3 \mathrm{a}$, suggesting overlapping gap MMN and P3a in the CI group (see Discussion).

Duration MMN amplitude was smaller and latency longer in the CI group than in the NH group (main effects of group, Table 3; Figure 2D). Duration MMN became larger by time only in the NH group and was at T2 significantly smaller in the CI group than in the $\mathrm{NH}$ group (interaction of group and time, Table 3; enlargement over time in the NH group, $p=0.001$; difference between groups at $\mathrm{T} 2, p=0.001)$. Importantly, at $\mathrm{T} 1$, the duration MMN was followed by $\mathrm{P} 3 \mathrm{a}$ in both groups. In contrast, at T2, the P3a was elicited only in the CI group (Table 2, Figure 2D). Therefore, we conducted the partial correlation analyses between the MMN and P3a amplitudes at T2 for the CI group. They correlated significantly (small change, $r_{p}=0.64$, $p=0.001$; medium change, $r_{p}=0.68, p=0.001$; large change, $\left.r_{p}=0.79, p<0.001\right)$. The MMN was smaller when the P3a was larger, suggesting a possible overlap of $\mathrm{MMN}$ and $\mathrm{P} 3 \mathrm{a}$ for the duration deviants in the CI group (see Discussion).

\section{P3a DEVELOPMENT WAS ENHANCED IN THE CI SINGERS}

Timbre MMN amplitude became smaller over time only in the CI singers (interaction of time and group, Table 4; post-hoc test, $p=0.005$ ) (Figures 3A, 4A, 5A). The interaction of time, age, and group was significant (Table 4 ).

Timbre P3a was earlier in the CI singers than in the CI nonsingers (main effect of group for latency, Table 4). Timbre P3a became larger over time in the CI singers but smaller and later over time in the CI non-singers and was significantly smaller and later in CI non-singers than in the CI singers at T2 (for the interactions of time and group, Table 4) (post hoc tests: in the CI singers, P3a amplitude increases, $p=0.017$; in the CI nonsingers, P3a amplitude decreases, $p=0.036$, P3a latency becomes longer, $p=0.001$; $\mathrm{P} 3 \mathrm{a}$ amplitude difference between groups at $\mathrm{T} 2, p=0.005, \mathrm{P} 3 \mathrm{a}$ latency difference between groups at $\mathrm{T} 2, p=$ 0.001) (Figures 3A, 4A, 5B).

Pitch MMN amplitude was larger in the CI non-singers than in the CI singers (main effect of group, Table 4). In the CI nonsingers alone, pitch $\mathrm{MMN}$ amplitude became larger over time especially for the large change, and was at T2 significantly larger

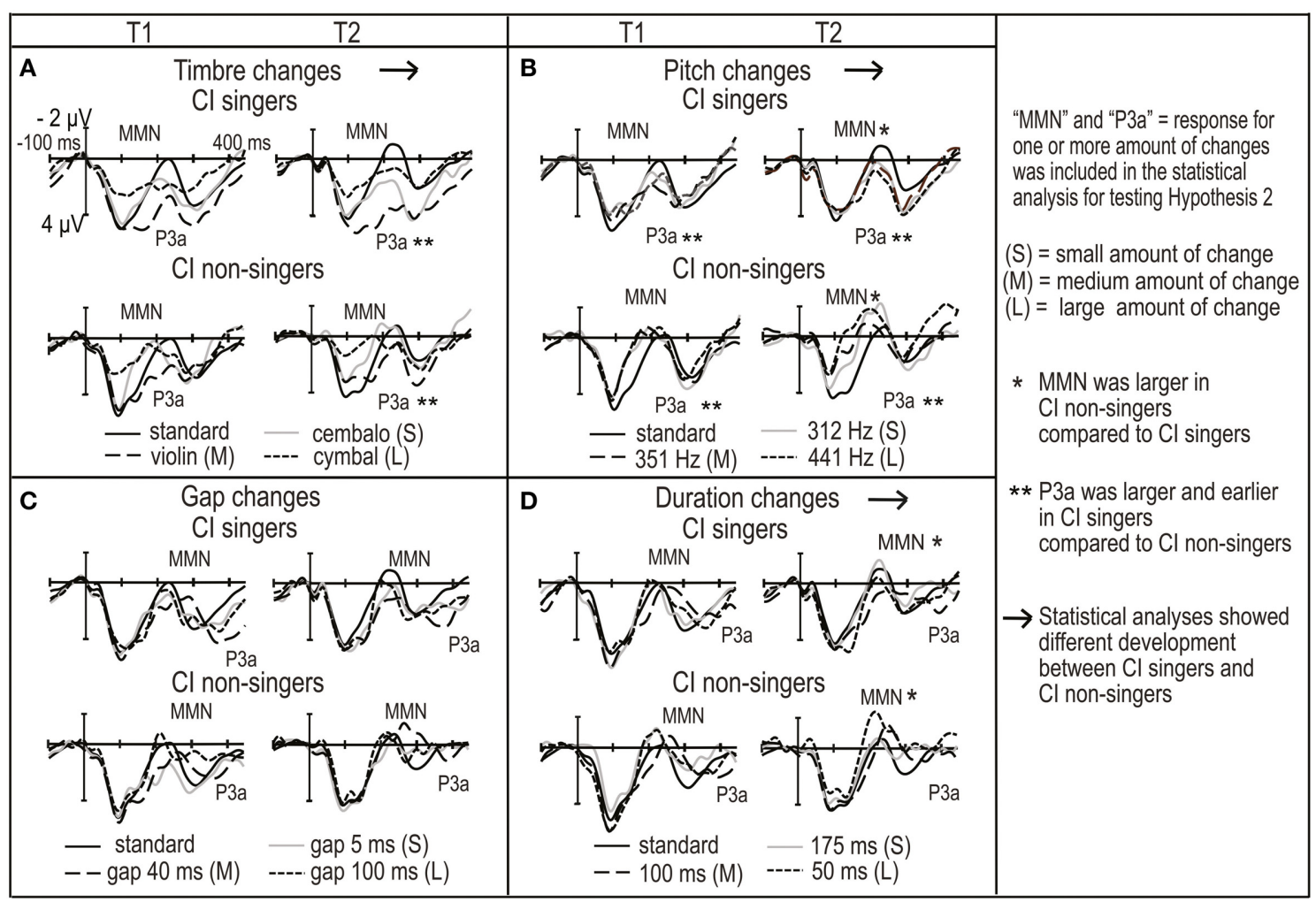

FIGURE 3 | The ERP ROI waveforms averaged across F3, Fz, F4, C3, Cz, and $\mathrm{C} 4$ electrodes for $\mathrm{Cl}$ singers and $\mathrm{Cl}$ non-singers for standard tones and for (A) timbre changes, (B) pitch changes, (C) gap changes and
(D) duration changes. The ERP waveforms are given for both time points of the measurements (T1 and T2 on the left and right in each panel, respectively). 


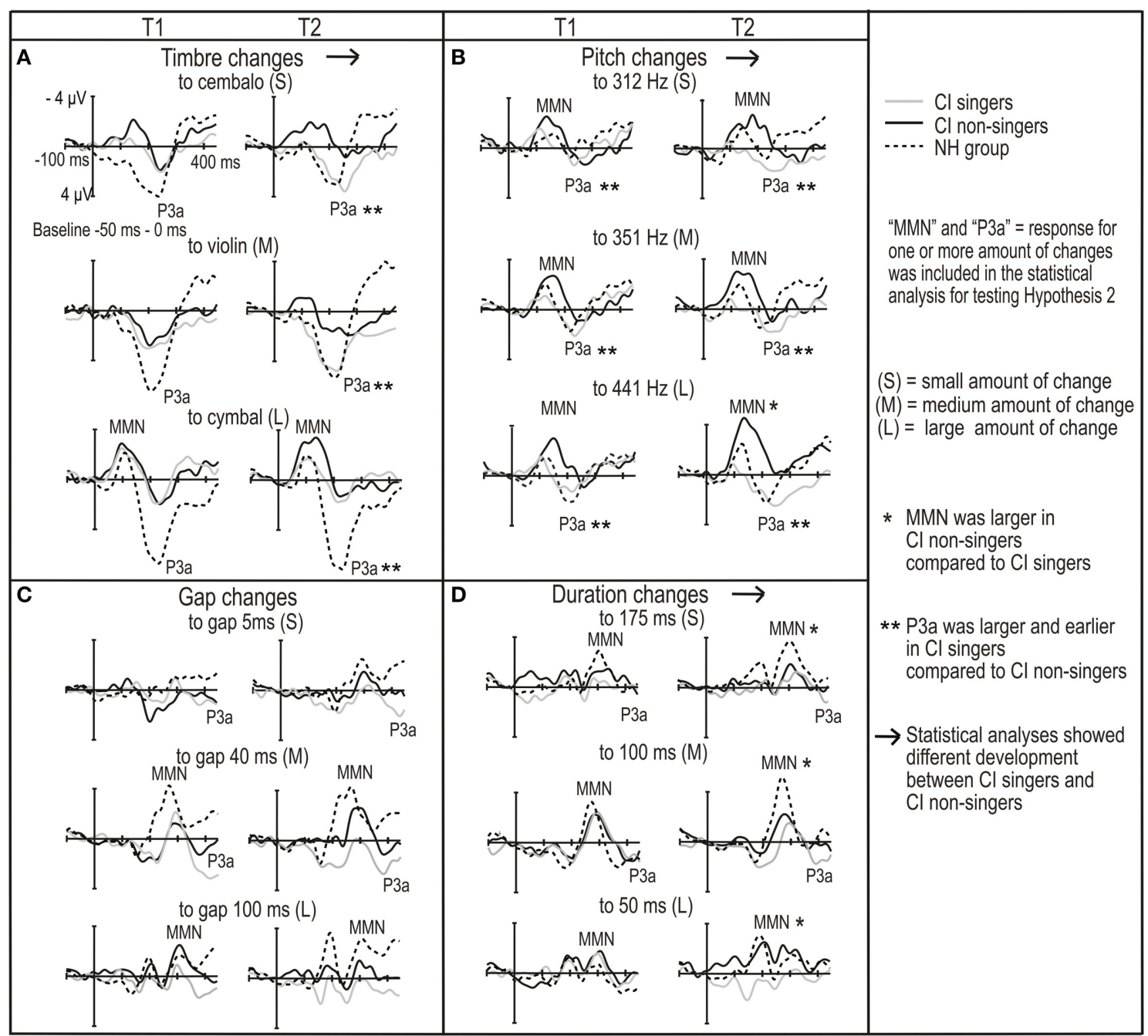

FIGURE 4 | The subtraction (deviant-standard) ROI waveforms averaged across F3, Fz, F4, C3, Cz and C4 electrodes for $\mathrm{NH}$ group, $\mathrm{Cl}$ singers and $\mathrm{Cl}$ non-singers for $(\mathrm{A})$ timbre changes, $(B)$ pitch changes,
(C) gap changes and (D) duration changes. The ERP waveforms are given for both time points of the measurements (T1 and T2 on the left and right in each panel, respectively). in them than in the CI singers (interaction of time and group, as well as time, amount and group, Table 4: post-hoc tests, enlargement of the MMN for the large pitch change, $p=0.004$; MMN amplitude difference between CI singers and CI non-singers for the large change at T2, $p<0.001$ ) (Figures 3B, 4B, 5A). The pitch $\mathrm{P} 3 \mathrm{a}$ of the CI non-singers, however, did not become larger over time with the pitch MMN. Importantly, the pitch P3a was larger and earlier in the CI singers than in the CI non-singers (main effects of group, Table 4, Figures 3B, 4B, 5B).

The differences between CI singers and CI non-singers in the $\mathrm{MMN}$ and $\mathrm{P} 3 \mathrm{a}$ for gaps were statistically not significant. However, the difference waves for medium and large gaps were completely positive at $\mathrm{T} 2$ in the $\mathrm{CI}$ singers but not in the $\mathrm{CI}$ non-singers (Figures 3C, 4C).

The CI singers had at T2 significantly smaller duration MMN than the CI non-singers (interaction of time and group, Table 4; post-hoc test, $p=0.049$ ) (Figures 3D, 4D and 5A). However, at $\mathrm{T} 2$ in the $\mathrm{CI}$ singers the difference wave was already positive in the time line of $\mathrm{MMN}$ especially for the large duration change (Figure 4D).

\section{DISCUSSION}

We compared CI children and normal-hearing ( $\mathrm{NH}$ ) children as well as the CI singers and the CI non-singers in their development of preattentive discrimination and attention shift toward changes in timbre, pitch, duration, and presence of temporal gaps in musical piano tones. The $4-13$ years old children participated in ERP recordings twice (at T1 and T2), in a period between 14 and 17 months. For the first time, we analyzed P3a responses for all change types. We found well-formed MMN and P3a in the $\mathrm{CI}$ group resembling those recorded in the $\mathrm{NH}$ group, which is in line with the previous findings on CI listeners (Kileny et al., 1997; Koelsch et al., 2004; Torppa et al., 2012). The CI and NH group as well as the CI singers and the CI non-singers differed in MMN and P3a development. Importantly, the P3a development was enhanced in the CI singers. 


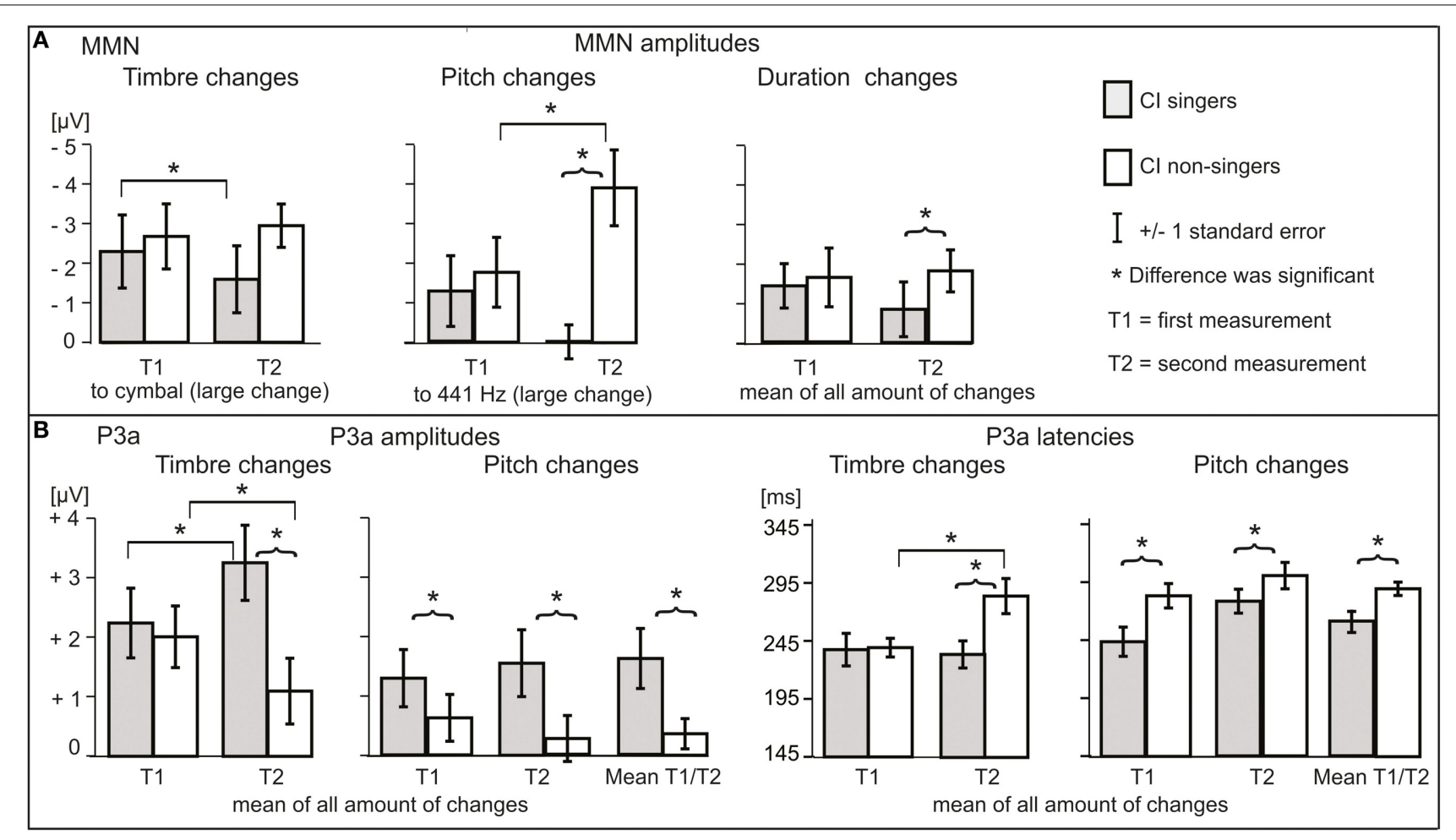

FIGURE 5 | The illustration of significant differences between $\mathrm{Cl}$ singers and $\mathrm{Cl}$ non-singers in (A) MMN and (B) P3a responses.

\section{THE DIFFERENCES BETWEEN CI AND NH GROUP}

As expected, timbre P3a was smaller in the CI group than in the NH group. This is in line with previous findings, showing difficulties in discrimination of timbre in CI recipients (see Introduction), and with results on degraded P3a in adult CI listeners (Koelsch et al., 2004; Nager et al., 2007). The present results on non-existent differences in pitch MMN and pitch P3a amplitudes between the $\mathrm{CI}$ and $\mathrm{NH}$ groups are also in line with Torppa et al. (2012). However, in the present study, we also found that the pitch P3a was later in the CI group. This indicates difficulties in discrimination of piano tone pitch in CI children, which is consistent with previous studies in CI recipients (see Introduction).

Intriguingly, the $\mathrm{CI}$ and $\mathrm{NH}$ groups differed in the development of MMN and P3a for rhythmic changes (for gaps and the changes in duration), which affects the interpretation of the results on $\mathrm{MMN}$ as follows. First, when the MMN for particular rhythmic change (gap, duration) was smaller in the CI group than in the $\mathrm{NH}$ group, then the $\mathrm{P} 3 \mathrm{a}$ for the rhythmic change was elicited only in the CI group. We also found that in the CI group, the P3a for these rhythmic changes overlapped with the MMN for the corresponding changes, i.e., the P3a peaked already at the assumed latency of the MMN (in the CI singers, see Section Differences Between CI Singers and CI Non-singers). Therefore, because the P3a of the CI group diminished their MMN, the CI vs. $\mathrm{NH}$ group comparisons on $\mathrm{MMN}$ for rhythmic changes were of little value. Second, in the NH group, when their duration P3a was very small or non-existent, their duration MMN was large.
This indicates that the changes in duration became less distractive for the NH group over time (see Wetzel et al., 2006 for the development of distraction and P3a over age in other context), and the lack of the overlap of P3a and MMN allowed their MMN to continue on growing as opposite to the CI group. This is the first time when both the MMN and P3a responses for gaps and duration changes in musical context are compared between CI and NH children. The important message for future research is that these child groups may develop differently on P3a, and this can affect the group comparisons on MMN.

It should be also noted that because the timbre and pitch changes at T2 still elicited P3a in the NH group, their P3a for these changes in musical context may diminish later than P3a for rhythmic changes. Moreover, the rapid development of duration MMN in the NH group could be a consequence of their development in the discrimination of musically relevant duration changes due to their exposure to music inside and outside of the home.

\section{TIMBRE P3a WITHOUT MMN}

We found timbre P3a without clear MMN for changes from piano to cembalo and violin. This was evident in the $\mathrm{NH}$ group and in part of the CI group, in the CI singers (Figure 4A). P3a without MMN has been found in many previous studies (Wetzel et al., 2006; Horváth et al., 2008; Koistinen et al., 2011, among others), however there seems to be no consensus on their interpretation. We showed for the first time that when the MMN was small or not visible in the subtraction curve, the amplitudes became more positive in the presumed time window of the $\mathrm{MMN}$ when the $\mathrm{P} 3 \mathrm{a}$ 
became larger. Thus, in the present study, the lack of MMN was evidently a consequence of the partly overlapping MMN and P3a responses. The response for change to cymbal elicited MMN and a large $\mathrm{P} 3 \mathrm{a}$ in all children; response to this change differed from the responses to other timbre changes, which may be explained as follows. The spectral difference between piano and cymbal tones is extremely large. This change may have activated fresh afferent neurons which, in turn, increased the amplitudes of the MMN and P3a. Instead, the spectral (acoustic) difference was rather small in the other timbre changes. Importantly, it seems that there is a distinction between large acoustic deviance processing and contextual information processing from a very early age. In the present study, the change to cymbal could have been processed as a large physical change leading to large MMN where the overlap of $\mathrm{P} 3 \mathrm{a}$ had small effect (the correlation was not significant between the MMN and P3a for cymbal change). In contrast, the change to cembalo and violin may have been processed as a contextual difference. This would be consistent with the proposal that the attention shift is sometimes a consequence of large physical difference and sometimes a consequence of contextual novelty (Kushnerenko et al., 2013).

\section{DIFFERENCES BETWEEN CI SINGERS AND CI NON-SINGERS}

The CI singers showed signs of enhanced development of P3a through all measured change types. First, the development of timbre P3a was enhanced in the CI singers as follows. At T2 their timbre P3a became comparable to that of the NH group for changes from piano to cembalo and violin (Figure 4A). The timbre P3a of the CI singers was significantly larger and earlier at T2 than in the CI non-singers, whose timbre P3a became smaller and later over time. Second, the pitch P3a was larger and earlier in the $\mathrm{CI}$ singers than in CI non-singers at T1 and T2. It is even possible that the non-significant difference between the CI and $\mathrm{NH}$ group in pitch $\mathrm{P} 3 \mathrm{a}$ amplitude is driven by the large $\mathrm{P} 3 \mathrm{a}$ responses of the CI singers. Third, we found in the CI singers positive, P3a-like responses without subsequent $\mathrm{MMN}$ at T2 for rhythm-related, medium or large changes (gaps and changes in duration), emerging at T2. Intriguingly, these early positive responses explain the smaller MMN in the CI singers compared to the CI non-singers for duration changes at T2, and may explain why the CI group had smaller duration MMN than NH group or why P3a coincided with MMN for rhythmic changes in the CI group. Moreover, the rapid emergence (after 14-17 months) of $\mathrm{P} 3 \mathrm{a}$ for rhythmic changes only in CI singers is consistent with the findings that P3a can emerge only after 5 days of training (Draganova et al., 2009). That they emerged to the largest changes is in turn consistent with the findings that P3a is strongly modulated by the amount of change (Wetzel et al., 2006; Koistinen et al., 2011, among others). Importantly, the enhanced production of rhythm of CI singers at T2 (Section Division of CI Group into CI Singers and CI Nonsingers) supports the interpretation that the positive responses are related to better perception. Further, the positive relation of singing to P3a responses is also consistent with the findings of Putkinen et al. (2013b), showing enhanced P3a responses for gap and duration changes in two to three year old $\mathrm{NH}$ children with a high amount of informal musical activities including singing.
The enhanced P3a development and the emergence of new, early P3a-like responses without $\mathrm{MMN}$ in the CI singers may reflect more accurate perception of changes than CI non-singers, at least in the present musical context (see previous section). Moreover, due to slow-rate, predictable singing, they perhaps discriminated or began to discriminate the changes better, further increasing distractability. The simultaneous enhancement of these factors may have led to the rapid enhancement or appearance of early P3a in the CI singers.

Interestingly, the pitch MMN became larger in CI non-singers without any evidence on enlargement of the pitch P3a. The P3a enlargement should have been expected because when MMN and P3a are both elicited, P3a becomes larger with larger MMN (Draganova et al., 2009). This may reflect development of preattentive discrimination without development of more attentional discrimination in the CI non-singers. This would show similarities with normal-hearing subjects who suffer from tone-deafness, also called congenital amusia, who have near-to-normal preattentive neural processing (MMN) of musical pitch incongruities even though they have limited conscious accuracy in such a task (Peretz et al., 2009). However, another interpretation might be that the neural networks for P3a develop less accurately in the CI non-singers than in the CI singers.

The neural network for P3a is distributed across frontal, parietal and temporal (auditory) cortical regions (Takahashi et al., 2013), suggesting functional connectivity between them. Interestingly, people suffering from amusia have degraded connections between frontal and temporal regions in their right hemisphere (Loui et al., 2009). Congenital deafness can also lead to degradation in white-matter volume in the auditory cortex and thus fewer afferent and efferent fibers (Emmorey et al., 2003). Because the increase in white-matter in association cortices, important for the maturation of auditory orienting, is already strong before the age of 8-12 months in normal-hearing children (Kushnerenko et al., 2013, for a review), missing auditory input even within the first years of life may harm the neural basis of attention shift toward sounds.

Importantly, normal-hearing singers have enhanced whitematter (anatomical) connectivity between frontal and temporal cortical regions (Halwani et al., 2011), and singing-based aphasia therapy seems to lead to similar enhancement (Wan et al., 2014). Based on the present results and the former evidence, singing could help CI children toward better functioning neural networks for auditory attention. This would be in line with the faster plastic changes in auditory and frontal areas in 6 years old children participating 15 months in musical training compared to other children (Hyde et al., 2009). Importantly, the plastic changes found by Hyde et al. (2009) were accompanied with enhanced development in auditory melodic and rhythmic discrimination.

\section{THE GENERAL IMPORTANCE OF THE PRESENT FINDINGS}

The present results suggest that when the ERP-responses of CI children are compared to $\mathrm{NH}$ children, one should pay attention to both MMN and P3a. First, the NH peers can be proceeding toward smaller $\mathrm{P} 3 \mathrm{a}$ when CI children are proceeding toward larger P3a. Second, the overlap of P3a with MMN can 
diminish the MMN. Third, the CI children who are exposed to multisensory musical activities, like singing, may differ from other CI children in the development of auditory attention shift. These aspects, if not taken into account, can affect the interpretations of the ERP responses.

The present study design cannot confirm the causality of singing in the development of attention shift. The enhanced P3a responses may be a consequence of some predispositions in CI singers which we could not find, and the CI singers may sing due to their better perceptual abilities or better neural networks for attention. However, the CI singers did not differ from CI non-singers in $\mathrm{CI}$ and hearing-related factors or socioeconomic or musical background (see Section Division of CI Group into CI Singers and CI Non-singers). Therefore, the present findings on P3a support the interpretation that singing enhances auditory attention and perception.

It is possible that more accurate perception of music with singing leads to enhanced enjoyment on music, which, in turn, helps children benefit more from music listening through the entire life span. Further, accurate processing of spectro-temporal changes, underlying the detection of changes in musical instrument timbre, is required for the perception of phonemes (Stevens, 1998; Patel, 2014). Perception of prosody, which is thought to assist perception and learning of spoken language, is better with good discrimination of pitch (Torppa et al., 2014) and musical rhythm (Hausen et al., 2013). It has already been suggested that improving music perception of CI listeners would enhance their speech perception (Drennan and Rubinstein, 2008). These findings are promising for the speech perception and spoken language learning of the CI singers.

Moreover, efficient attention shifts are necessary in order to process rapidly changing auditory scenes like in traffic, or in schools, day cares and other places where the attention should be directed quickly toward important sounds. It is also necessary for the further cognitive processing of incoming sounds (Friedman et al., 2001), and this enhanced attention shift toward sound changes may help the CI singers in their everyday life.

\section{THE LIMITATIONS OF THE PRESENT STUDY}

The present study design includes several types and magnitudes of changes in two groups of children recorded twice. This led us to conduct large amount of statistical analyses, but we corrected for multiple testing only in the post-hoc tests. This might lead to type 1 errors, i.e., some connections could be significant by chance. However, the results show a coherent general enhancement of P3a in CI singers. In addition, using statistical correction for reducing the responses taken into analyses would have increased the type 2 errors (missing significant responses) due to singing-related variation in the timing of the responses. For example, at T2 CI non-singers had pitch MMN in the latency window for the amplitude analyses for pitch P3a, and the CI singers had intensity, duration and gap P3a in the time window of the amplitude analyses for MMN, which inevitably reduces the significance of these responses. Therefore, we are assured that the best solution was to use relatively liberal correction procedures.

\section{CONCLUSIONS}

Based on current MMN and P3a findings, singing may enhance perceptual and attentional functions related to music and, possibly, sounds in general. We found an interplay between development of MMN and P3a responses, hearing status, and singing of CI children. The present findings are novel especially because they are the first showing enhanced $\mathrm{P} 3$ a responses related to singing of CI children. Even though the present study cannot show direct causality of singing to brain development and enhanced auditory processing, these findings should boost future studies on the effects of singing, especially on attention toward sounds. Singing requires no expensive musical instruments or formal instruction and is readily available for everyone. From both the economical and ethical perspectives, the present findings may be most important for the rehabilitation and quality of life of CI children. It also seems that despite of their difficulties in singing in tune (Nakata et al., 2006; Xu et al., 2009), children with CIs should be given opportunities and be encouraged to sing. Still, an important goal of future studies is to confirm the causality of the connections between singing and the advanced auditory perception and attention.

\section{ACKNOWLEDGMENTS}

This study was financially supported by the Signe and Ane Gyllenberg Foundation, the Finnish Concordia Fund, the Ella and Georg Ehrnrooth Foundation, National doctoral program Langnet and the Emil Aaltonen Foundation. We thank Prof. Andrew Faulkner, Prof. Martti Vainio, Prof. Kimmo Alho, TOD Christine Rocca, Lindfors Foundation (Eila Lonka and Helena Ahti), Finnish Audiological Society, the personnel of Departments of Audiology in Helsinki, Turku, Tampere and Kuopio University Hospitals and Cognitive Brain Research Unit (especially Tanja Linnavalli, Johannes Pykäläinen, Hannu Loimo, Emma Salo, Tommi Makkonen, Gabriel Nathan Friedman and Caitlin Dawson) involved in this study, and most importantly, the parents and children for their participation.

\section{SUPPLEMENTARY MATERIAL}

The Supplementary Material for this article can be found online at: http://www.frontiersin.org/journal/10.3389/fpsyg. 2014.01389/abstract

\section{REFERENCES}

Akaike, H. (1974). A new look at the statistical model identification. IEEE Trans. Autom. Control 19, 716-723. doi: 10.1109/TAC.1974.11 00705

Bahrick, L. E., and Lickliter, R. (2000). Intersensory redundancy guides attentional selectivity and perceptual learning in infancy. Dev. Psychol. 36, 190-201. doi: 10.1037/0012-1649.36.2.190

Bryk, S. W., and Raudenbush, A. S. (2002). Hierarchical Linear Models: Applications and Data Analysis Methods, 3rd Edn. Thousand Oaks, CA: Sage Publications.

Chen, J. K. C., Chuang, A. Y. C., McMahon, C., Hsieh, J. C., Tung, T. H., and $\mathrm{Li}, \mathrm{L}$. P. (2010). Music training improves pitch perception in prelingually deafened children with cochlear implants. Pediatrics 125, e793-e800. doi: 10.1542/peds.2008-3620

Chobert, J., François, C., Velay, J.-L., and Besson, M. (2012). Twelve months of active musical training in 8- to 10 -year-old children enhances the preattentive processing of syllabic duration and voice onset time. Cereb. Cortex 24, 956-967. doi: $10.1093 /$ cercor/bhs377 
Delorme, A., and Makeig, S. (2004). EEGLAB: an open source toolbox for analysis of single-trial EEG dynamics including independent component analysis. J. Neurosci. Methods 134, 9-21. doi: 10.1016/j.jneumeth.2003.10.009

Draganova, R., Wollbrink, A., Schulz, M., Okamoto, H., and Pantev, C. (2009). Modulation of auditory evoked responses to spectral and temporal changes by behavioral discrimination training. BMC Neurosci. 10:143. doi: 10.1186/14712202-10-143

Drennan, W. R., and Rubinstein, J. T. (2008). Music perception in cochlear implant users and its relationship with psychophysical capabilities. J. Rehabil. Res. Dev. 45, 779-790. doi: 10.1682/JRRD.2007.08.0118

Emmorey, K., Allen, J. S., Bruss, J., Schenker, N., and Damasio, H. (2003). A morphometric analysis of auditory brain regions in congenitally deaf adults. Proc. Natl. Acad. Sci. U.S.A. 100, 10049-10054. doi: 10.1073/pnas.17301 69100

Escera, C., Alho, K., Winkler, I., and Näätänen, R. (1998). Neural mechanisms of involuntary attention to acoustic novelty and change. J. Cogn. Neurosci. 10, 590-604. doi: 10.1162/089892998562997

Fagan, M. K., and Pisoni, D. B. (2009). Perspectives on multisensory experience and cognitive development in infants with cochlear implants. Scand. J. Psychol. 50, 457-462. doi: 10.1111/j.1467-9450.2009.00743.x

Friedman, D., Cycowicz, Y. M., and Gaeta, H. (2001). The novelty P3: an event-related brain potential (ERP) sign of the brain's evaluation of novelty. Neurosci. Biobehav. Rev. 25, 355-373. doi: 10.1016/S0149-7634(01)00 019-7

Fujioka, T., Ross, B., Kakigi, R., Pantev, C., and Trainor, L. J. (2006). One year of musical training affects development of auditory cortical-evoked fields in young children. Brain 129, 2593-2608. doi: 10.1093/brain/awl247

Galvin, J. J. III., Fu, Q. J., and Shannon, R. V. (2009). Melodic contour identification and music perception by cochlear implant users. Ann. N.Y. Acad. Sci. 1169, 518-533. doi: 10.1111/j.1749-6632.2009.04551.x

Gfeller, K., Knutson, J. F., Woodworth, G., Witt, S., and DeBus, B. (1998). Timbral recognition and appraisal by adult cochlear implant users and normal-hearing adults. J. Am. Acad. Audiol. 9, 1-19.

Gilley, P. M., Sharma, A., Dorman, M., Finley, C. C., Panch, A. S., and Martin, K. (2006). Minimization of cochlear implant stimulus artifact in cortical auditory evoked potentials. Clin. Neurophysiol. 117, 1772-1782. doi: 10.1016/j.clinph.2006.04.018

Halwani, G. F., Loui, P., Rüber, T., and Schlaug, G. (2011). Effects of practice and experience on the arcuate fasciculus: comparing singers, instrumentalists, and non-musicians. Front. Psychol. 2:156. doi: 10.3389/fpsyg.2011.00156

Hausen, M., Torppa, R., Salmela, V. R., Vainio, M., and Särkäm,ö, T. (2013). Music and speech prosody: a common rhythm. Front. Psychol. 4:566. doi: 10.3389/fpsyg.2013.00566

Herholz, S. J., and Zatorre, R. J. (2012). Musical training as a framework for brain plasticity: Behavior, function, and structure. Neuron 76, 486-502. doi: 10.1016/j.neuron.2012.10.011

Horváth, J., Winkler, I., and Bendixen, A. (2008). Do N1/MMN, P3a, and RON form a strongly coupled chain reflecting the three stages of auditory distraction? Biol. Psychol. 79, 139-147. doi: 10.1016/j.biopsycho.2008.04.001

Houston, D. M., and Bergeson, T. R. (2014). Hearing versus listening: attention to speech and its role in language acquisition in deaf infants with cochlear implants. Lingua 139, 10-25. doi: 10.1016/j.lingua.2013.08.001

Houston, D. M., Pisoni, D. B., Kirk, K. I., Ying, E. A., and Miyamoto, R. T. (2003). Speech perception skills of deaf infants following cochlear implantation: a first report. Int. J. Pediatr. Otorhinolaryngol. 67, 479-495. doi: 10.1016/S01655876(03)00005-3

Hsiao, F., and Gfeller, K. (2012). Music perception of cochlear implant recipients with implications for music instruction: a review of literature. Uppdate Appl. Res. Music Educ. 30, 5-10. doi: 10.1177/8755123312437050

Hyde, K. L., Lerch, J., Norton, A., Forgeard, M., Winner, E., Evans, A. C., et al. (2009). Musical training shapes structural brain development. J. Neurosci. 29, 3019-3025. doi: 10.1523/JNEUROSCI.5118-08.2009

Kelly, A. S., Purdy, S. C., and Thorne, P. R. (2005). Electrophysiological and speech perception measures of auditory processing in experienced adult cochlear implant users. Clin. Neurophysiol. 116, 1235-1246. doi: 10.1016/j.clinph.2005.02.011

Kileny, P., Boerst, A., and Zwolan, T. (1997). Cognitive evoked potentials to speech and tonal stimuli in children with implants. Otolaryngol. Head Neck Surg. 117, 161-169. doi: 10.1016/S0194-5998(97)70169-4
Koelsch, S., Wittfoth, M., Wolf, A., Muller, J., and Hahne, A. (2004). Music perception in cochlear implant users: an event-related potential study. Clin. Neurophysiol. 115, 966-972. doi: 10.1016/j.clinph.2003.11.032

Koistinen, S., Rinne, T., Cederström, S., and Alho, K. (2011). Effects of significance of auditory location changes on event related brain potentials and pitch discrimination performance. Brain Res. 1427, 44-53. doi: 10.1016/j.brainres.2011.09.034

Kral, A., and Sharma, A. (2012). Developmental neuroplasticity after cochlear implantation. Trends. Neurosci. 35, 111-122. doi: 10.1016/j.tins.2011.09.004

Kronenberger, W. K., Beer, J., Castellanos, I., Pisoni, D. B., and Miyamoto, R. T. (2014). Neurocognitive risk in children with cochlear implants. JAMA Otolaryngol. Head Neck Surg. 140, 608-615. doi: 10.1001/jamaoto. 2014.757

Kujala, T., Tervaniemi, M., and Schröger, E. (2007). The mismatch negativity in cognitive and clinical neuroscience: theoretical and methodological considerations. Biol. Psychol. 74, 1-19. doi: 10.1016/j.biopsycho.2006.06.001

Kushnerenko, E. V., Van den Bergh, B. R. H., and Winkler, I. (2013). Separating acoustic deviance from novelty during the first year of life: a review of event-related potential evidence. Front. Psychol. 4:595. doi: 10.3389/fpsyg.2013. 00595

Limb, C. J., and Roy, A. T. (2014). Technological, biological, and acoustical constraints to music perception in cochlear implant users. Hear. Res. 308, 13-26. doi: 10.1016/j.heares.2013.04.009

Lonka, E., Relander-Syrjänen, K., Johansson, R., Näätänen, R., Alho, K., and Kujala, T. (2013). The mismatch negativity (MMN) brain response to sound frequency changes in adult cochlear implant recipients: a follow-up study. Acta Otolaryngol. 133, 853-857. doi: 10.3109/00016489.2013.780293

Loui, P., Alsop, D., and Schlaug, G. (2009). Tone deafness: a new disconnection syndrome? J. Neurosci. 29, 10215-10220. doi: 10.1523/JNEUROSCI.1701-09.2009

Lovio, R., Pakarinen, S., Huotilainen, M., Alku, P., Silvennoinen, S., Näätänen, R., et al. (2009). Auditory discrimination profiles of speech sound changes in 6-year-old children as determined with the multi-feature MMN paradigm. Clin. Neurophysiol. 120, 916-921. doi: 10.1016/j.clinph.2009.03.010

Makeig, S., Debener, S., Onton, J., and Delorme, A. (2004). Mining event-related brain dynamics. Trends Cogn. Sci. 8, 204-210. doi: 10.1016/j.tics.2004.03.008

Margulis, E. H. (2008). Neuroscience, the food of musical culture? Rev. Gen. Psychol. 12, 159-169. doi: 10.1037/1089-2680.12.2.159

McDermott, H. J. (2004). Music perception with cochlear implants: a review. Trends Amplif. 8, 49-82. doi: 10.1177/108471380400800203

Moore, B. C. (2003). Coding of sounds in the auditory system and its relevance to signal processing and coding in cochlear implants. Otol. Neurotol. 24, 243-254. doi: 10.1097/00129492-200303000-00019

Moore, J. K., and Linthicum, F. H. (2007). The human auditory system: a timeline of development. Int. J. Audiol. 46, 460-478. doi: 10.1080/14992020701383019

Moreno, S., Marques, C., Santos, A., Santos, M., Castro, S. L., and Besson, M. (2009). Musical training influences linguistic abilities in 8-year-old children: more evidence for brain plasticity. Cereb. Cortex 19, 712-723. doi: 10.1093/cercor/bhn 120

Näätänen, R., Gaillard, A., and Mäntysalo, S. (1978). Early selective attention effect reinterpreted. Acta Psychol. 42, 313-329. doi: 10.1016/0001-6918(78)90006-9

Näätänen, R., Pakarinen, S., Rinne, T., and Takegata, R. (2004). The mismatch negativity (MMN): towards the optimal paradigm. Clin. Neurophysiol. 115, 140-144. doi: 10.1016/j.clinph.2003.04.001

Nager, W., Münte, T. F., Bohrer, I., Lenarz, T., Dengler, R., Möbes, J., et al. (2007). Automatic and attentive processing of sounds in cochlear implant patients electrophysiological evidence. Restor. Neurol. Neurosci. 25, 391-396.

Nakata, T., and Trehub, S. E. (2004). Infants' responsiveness to maternal speech and singing. Infant Behav. Dev. 27, 455-464. doi: 10.1016/j.infbeh.2004.03.002

Nakata, T., Trehub, S. E., Mitani, C., and Kanda, Y. (2006). Pitch and timing in the songs of deaf children with cochlear implants. Music Percept. 24, 147-154. doi: 10.1525/mp.2006.24.2.147

Patel, A. D. (2014). Can nonlinguistic musical training change the way the brain processes speech? The expanded OPERA hypothesis. Hear. Res. 308, 98-108. doi: 10.1016/j.heares.2013.08.011

Peretz, I., Brattico, E., Järvenpää, M., and Tervaniemi, M. (2009). The amusic brain: in tune, out of key, and unaware. Brain 132, 1277-1286. doi: 10.1093/brain/awp055

Petersen, B., Mortensen, M. V., Hansen, M., and Vuust, P. (2012). Singing in the key of life - a pilot study on effects of musical ear training 
after cochlear implantation. Psychomusicology 22, 134-151. doi: 10.1037/ a0031140

Ponton, C. W., Eggermont, J. J., Waring, M. D., Kwong, B., Cunningham, J., and Trautwein, P. (2000). Maturation of the mismatch negativity: effects of profound deafness and cochlear implant use. Audiol. Neurotol. 5, 167-185. doi: $10.1159 / 000013878$

Putkinen, V., Saarikivi, K., and Tervaniemi, M. (2013a). Do informal musical activities shape auditory skill development in preschool-age children? Front. Psychol. 4:572. doi: 10.3389/fpsyg.2013.00572

Putkinen, V., Tervaniemi, M., and Huotilainen, M. (2013b). Informal musical activities are linked to auditory discrimination and attention in 2-3-year-old children: an event-related potential study. Eur. J. Neurosci. 37, 654-661. doi: 10.1111/ejn.12049

Putkinen, V., Tervaniemi, M., Saarikivi, K., Ojala, P., and Huotilainen, M. (2014). Enhanced development of auditory change detection in musically trained school-aged children: a longitudinal event-related potential study. Dev. Sci. 17, 282-297. doi: 10.1111/desc.12109

Rocca, C. (2012). A different musical perspective: improving outcomes in music through habilitation, education, and training for children with cochlear implants. Semin. Hear. 33, 425-433. doi: 10.1055/s-0032-1329229

Rochette, F., Moussard, A., and Bigand, E. (2014). Music lessons improve auditory perceptual and cognitive performance in deaf children. Front. Hum. Neurosci. 8:488. doi: 10.3389/fnhum.2014.00488

Ronkainen, R. (2011). Enhancing listening and imitation skills in children with cochlear implants: the use of multimodal resources in speech and language therapy. J. Interact. Res. Commun. Disord. 2, 245-269. doi: 10.1558/jircd.v2i2.245

Saarikallio, S. (2010). Music as emotional self-regulation throughout adulthood Psychol. Music. 39, 307-327. doi: 10.1177/0305735610374894

Sandmann, P., Kegel, A., Eichele, T., Dillier, N., Lai, W., Bendixen, A., et al. (2010). Neurophysiological evidence of impaired musical sound perception in cochlear-implant users. Clin. Neurophysiol. 121, 2070-2082. doi: 10.1016/j.clinph.2010.04.032

Särkämö, T., Tervaniemi, M., Laitinen, S., Numminen, A., Kurki, M., Johnson, J. K., et al. (2014). Cognitive, emotional, and social benefits of regular musical activities in early dementia: randomized controlled study. Gerontologist 54, 634-650. doi: 10.1093/geront/gnt100

Schwarz, G. E. (1978). Estimating the dimension of a model. Ann. Stat. 6, 461-464. doi: $10.1214 /$ aos/1176344136

Singer, J., and Wilett, J. (2003). Applied Longitudinal Data Analysis: Modeling Change and Event Occurrence. New York, NY: Oxford University Press. doi: 10.1093/acprof:oso/9780195152968.001.0001

Stevens, K. N. (1998). Acoustic Phonetics. Cambridge, MA; London: The MMIT press.

Strait, D. L., Parbery-Clark, A., Hittner, E., and Kraus, N. (2012). Musical training during early childhood enhances the neural encoding of speech in noise. Brain Lang. 123, 191-201. doi: 10.1016/j.bandl.2012.09.001

Takahashi, H., Rissling, A. J., Pascual-Marqui, R., Kirihara, K., Pela, M., Sprock, J., et al. (2013). Neural substrates of normal and impaired preattentive sensory discrimination in large cohorts of nonpsychiatric subjects and schizophrenia patients as indexed by MMN and P3a change detection responses. Neuroimage 66, 594-603. doi: 10.1016/j.neuroimage.2012.09.074

Timm, L., Agrawal, D. C., Viola, F., Sandmann, P., Debener, S., Büchner, A., et al. (2012). Temporal feature perception in cochlear implant users. PLoS ONE 7:e45375. doi: 10.1371/journal.pone.0045375

Timm, L., Vuust, P., Brattico, E., Agrawal, D., Debener, S., Büchner, A., et al. (2014). Residual neural processing of musical sound features in adult cochlear implant users. Front. Hum. Neurosci. 8:181. doi: 10.3389/fnhum.2014.00181

Torppa, R., Faulkner, A., Huotilainen, M., Järvikivi, J., Lipsanen, J., Laasonen, M., et al. (2014). The perception of prosody and associated auditory cues in earlyimplanted children; the role of auditory working memory and musical activities. Int. J. Audiol. 53, 182-191. doi: 10.3109/14992027.2013.872302

Torppa, R., Salo, E., Makkonen, T., Loimo, H., Pykäläinen, J., Lipsanen, J., et al. (2012). Cortical processing of musical sounds in children with cochlear implants. Clin. Neurophysiol. 123, 1966-1979. doi: 10.1016/j.clinph.2012. 03.008

Tremblay, K., Kraus, N., and McGee, T. (1998). The time course of auditory perceptual learning: neurophysiological changes during speech-sound training. Neuroreport 8, 3557-3560.

Uther, M., Kujala, A., Huotilainen, M., Shtyrov, Y., and Näätänen, R. (2006). Training in Morse code enhances involuntary attentional switching to acoustic frequency: evidence from ERPs. Brain Res. 1073, 417-424. doi: 10.1016/j.brainres.2005.12.047

van Zuijen, T. L., Simoens, V. L., Paavilainen, P., Näätänen, R., and Tervaniemi, M. (2006). Implicit, intuitive, and explicit knowledge of abstract regularities in a sound sequence: an event-related brain potential study. J. Cogn. Neurosci. 18, 1292-1303. doi: 10.1162/jocn.2006.18.8.1292

Wan, C. Y., Zheng, X., Marchina, S., Norton, A., and Schlaug, G. (2014). Intensive therapy induces contralateral white matter changes in chronic stroke patients with Broca's aphasia. Brain Lang. 136C, 1-7. doi: 10.1016/j.bandl.2014. 03.011

West, B. T. (2009). Analyzing longitudinal data with the Linear Mixed Models procedure in SPSS. Eval. Health Prof. 32, 207-228. doi: 10.1177/01632787093 38554

Wetzel, N., Widmann, A., Berti, S., and Schröger, E. (2006). The development of involuntary and voluntary attention from childhood to adulthood: a combined behavioral and event-related potential study. Clin. Neurophysiol. 117, 2191-2203. doi: 10.1016/j.clinph.2006.06.717

Wild, J., Yusuf, A., Wilson, D. E., Peelle, J. E., Davis, M. H., and Johnsrude, I. S. (2012). Effortful listening: the processing of degraded speech depends critically on attention. J. Neurosci. 32, 14010-14021. doi: 10.1523/JNEUROSCI.152812.2012

Winkler, I., Tervaniemi, M., Schröger, E., Wolff, C., and Näätänen, R. (1998) Preattentive processing of auditory spatial information in humans. Neurosci. Lett. 242, 49-52. doi: 10.1016/S0304-3940(98)00022-6

Xu, L., Zhou, N., Chen, X., Li, Y., Schultz, H. M., Zhao, X., et al. (2009). Vocal singing by prelingually-deafened children with cochlear implants. Hear. Res. 255, 129-234. doi: 10.1016/j.heares.2009.06.011

Yabe, H., Saito, F., and Fukushima, Y. (1993). Median method for detecting endogenous event-related brain potentials. Electroencephalogr. Clin. Neurophysiol. 87, 403-407. doi: 10.1016/0013-4694(93)90154-N

Yucel, E., Sennaroglu, G., and Belgin, E. (2009). The family oriented musical training for children with cochlear implants: speech and musical perception results of two year follow-up. Int. J. Pediatr. Otorhinolaryngol. 73, 1043-1052. doi: 10.1016/j.ijporl.2009.04.009

Zatorre, R. J., and Salimpoor, V. N. (2013). From perception to pleasure: Music and its neural substrates. Proc. Natl. Acad. Sci. U.S.A. 18(Suppl. 2), 10430-10437. doi: $10.1073 /$ pnas. 1301228110

Conflict of Interest Statement: The authors declare that the research was conducted in the absence of any commercial or financial relationships that could be construed as a potential conflict of interest.

Received: 26 May 2014; accepted: 13 November 2014; published online: 10 December 2014.

Citation: Torppa R, Huotilainen M, Leminen M, Lipsanen J and Tervaniemi M (2014) Interplay between singing and cortical processing of music: a longitudinal study in children with cochlear implants. Front. Psychol. 5:1389. doi: 10.3389/fpsyg.2014.01389 This article was submitted to Auditory Cognitive Neuroscience, a section of the journal Frontiers in Psychology.

Copyright (c) 2014 Torppa, Huotilainen, Leminen, Lipsanen and Tervaniemi. This is an open-access article distributed under the terms of the Creative Commons Attribution License (CC BY). The use, distribution or reproduction in other forums is permitted, provided the original author (s) or licensor are credited and that the original publication in this journal is cited, in accordance with accepted academic practice. No use, distribution or reproduction is permitted which does not comply with these terms. 